\title{
Ligand-independent role of ErbB3 in endocytic recycling
}

\author{
Ana Rosa Saez-Ibanez $^{1,2,3}$, Julia M. Scheffler ${ }^{1,4 \#}$, Takeshi Terabayashi ${ }^{1,5 \#}$, Nina Daubel ${ }^{6}$, Taija \\ Makinen $^{6}$, Olof Idevall-Hagren ${ }^{7}$, Ingvar Ferby ${ }^{1,2 *}$
}

${ }^{1}$ Ludwig Institute for Cancer Research, Science for Life Laboratory, Uppsala, Sweden

2

Department of Medical Biochemistry and Microbiology, Science for Life Laboratory, Uppsala

University, Uppsala, Sweden

${ }^{3}$ Current address: Department of Developmental and Molecular Biology, Albert Einstein College of

Medicine, Bronx and Department of Medicine, Division of Hematology and Medical Oncology, Icahn

School of Medicine, Tisch Cancer Institute at Mount Sinai, New York, USA

4 Current address: Department of Rheumatology and Inflammation Research, Gothenburg University,

Gothenburg, Sweden

${ }^{5}$ Current address: Department of Pharmacology, Oita University, Faculty of Medicine, Oita, Japan

${ }^{6}$ Department of Immunology, Genetics and Pathology, Uppsala University, Uppsala, Sweden

7 Department of Medical Cell Biology, Uppsala University, Uppsala, Sweden

\# ${ }_{\text {these authors contributed equally }}$

*To whom correspondence should be addressed: Ingvar Ferby, e-mail:ingvar.ferby@imbim.uu.se

Running title: Integral role of ErbB3 in the endocytic recycling machinery

\section{Key words:}

ErbB3, endocytic recycling, integrin $\beta 1$, rabaptin-5, GGA3, Rab4, endosome 


\begin{abstract}
Activated receptor tyrosine kinases (RTKs) are important cargo of the endocytic trafficking, yet to what extent RTKs play a role in endocytic trafficking processes per se, remains unclear. Here we show that the ErbB3 receptor, frequently overexpressed in invasive cancers, sorts endocytosed cargo including $\beta 1$ integrins and the transferrin receptor (TfR) for endocytic recycling in breast epithelial cells, in a manner that does not require ligand-induced ErbB3 signalling. Loss of ErbB3 abrogates recycling of $\beta 1$ integrins, likely from a Rab4-positive compartment, and redirects it towards lysosomal degradation. Consequently, delivery of $\beta 1$ integrins to the leading front of migrating sheets of epithelial cells is impaired and collective migration compromised upon loss of ErbB3. Mechanistically, ErbB3 interacts with the endosomal adaptors GGA3 and Rabaptin5 facilitating assembly of an Arf6-GGA3-Rabaptin5 endosomal sorting complex, to promote recycling of cargo such as integrins and TfR. Taken together, our results show that ErbB3 is an integral part of the endosomal trafficking machinery, provoking the notion that RTKs might play yet unrecognised roles in vesicular trafficking.
\end{abstract}

\title{
Introduction
}

The Epidermal growth factor receptor (EGFR) family of receptor tyrosine kinases (RTKs), including EGFR, ErbB2, ErbB3 and ErbB4, form homo- or heterodimers and play key roles in epithelial development and homeostasis while their deregulation contributes to most epithelial cancers ${ }^{1}$. Upon ligand-binding the RTK dimers are activated, leading to recruitment of signalling molecules that initiate intracellular signalling cascades. ErbB3 has limited kinase activity but signal as heterodimers with other members of the EGFR family and accumulating evidence highlight its potent oncogenic activity. In particular, ErbB3 has been linked to the migratory behaviour and metastasis of cancer cells $^{2-5}$. ErbB3 is also responsible for acquired resistance to anti-EGFR and -ErbB2 therapies, and thus an intensely pursued therapeutic target (reviewed in ${ }^{6}$ ).

Ligand-binding to RTKs triggers receptor internalisation and trafficking within the cells which determines strength, duration and spatial distribution of EGFR signals ${ }^{7-11}$. RTKs, including EGFRs are internalised through clathrin-dependent and -independent mechanisms, converging on the delivery of the receptors to early endosomes ${ }^{7}$. Thereafter, the receptors are either diverted towards protein degradation via late endosomes/lysosomes or recycled back to the plasma membrane. Endocytic recycling can occur via different vesicular compartments which require the involvement of distinct 
Rab GTPases and other trafficking effectors. Among them, the Rab4-dependent "short-loop" and the Rab11-dependent "long-loop" recycling constitute two major pathways followed by RTKs and other transmembrane proteins, including integrins ${ }^{12,13}$.

Recently, the ubiquitously expressed GGA1-3 proteins, which are clathrin adaptors dependent on small GTPases of the Arf family, have been found to regulate Rab4 or -11-dependent endosomal recycling of $\beta 1$ integrins (GGA2, GGA3), Transferrin receptor (TfR) (GGA1), Met RTK and Ret RTK $(\text { GGA3 })^{14-20}$. GGA proteins contain several functional domains that, for example, bind accessory proteins that modulate membrane trafficking such as Rabaptin5 ${ }^{18,21,22}$. Rabaptin5 and the closely related family member rabaptin4 are effectors of both Rab4 and Rab5 ${ }^{23-25}$. Rabaptins promote fusion of vesicles to early endosomes and are additionally thought to cooperatively link Rab5- and Rab4containing microdomains on early endosomes to coordinate the coupling between these domains ${ }^{26}$ thereby regulating sorting of cargo including integrin $\beta 3$ and TfR into recycling endosomes ${ }^{26-28}$.

Here, we studied the role ErbB3 in vesicular trafficking and found that it constitutes an integral component of the endocytic recycling machinery that is required for recycling of $\beta 1$ integrins and the TfR. ErbB3 associates with and promotes assembly of the Arf6-GGA3-rabaptin5 adaptor complex by stabilising the GGA3 and Rabaptin5 proteins.

\section{Results}

\section{ErbB3 is required for endocytic recycling of $\beta 1$ integrins}

ErbB3 har been found to continuously endocytose and recycle ${ }^{30,31}$ back to the plasma membrane in a manner that does not require ligand stimulation or other members of the EGFR family ${ }^{31}$. We wondered if endosomal trafficking of ErbB3 might be co-ordinated with, or even regulate endocytic trafficking of cargo such as $\beta 1$ integrins that also continuously internalise and recycle back to the plasma membrane to support cell motility. Firstly, we asked if ErbB3 co-localises with internalised $\beta 1$ integrins on a breast epithelial cell-line (MCF7) that expresses high detectable levels of endogenous ErbB3. Briefly, integrin $\beta 1$ was labelled on ice with an Alexa488-conjugated antibody, and allowed to internalise for 15 minutes at $37^{\circ} \mathrm{C}$, prior to fixation and immunolabeling of endogenous ErbB3. Confocal microscopy showed that ErbB3 and traced integrin $\beta 1$ co-localised on intracellular structures likely to be endosomes based on their estimated size $(0.5-2 \mu \mathrm{m})$, as well as in filopodia (Fig. 1a-c). The degree of co-localisation on putative endosomes was determined from deconvolved confocal images by measuring enrichment of integrin $\beta 1$ on ErbB3-positive putative endosomes, relative to their adjacent surrounding (Fig. 1d). 32\% of the ErbB3-positive endosomal structures showed prominent (>100\%) enrichment of integrin $\beta 1$ (Fig. 1d). 
We next investigated the putative role of ErbB3 in endocytic trafficking of $\beta 1$ integrins by conducting a recycling assay as outlined in figure 1e. Briefly, the cell-surface pools of $\beta 1$ integrins were labelled with Alexa488-conjugated antibody on ice, prior to 15 minutes incubation at $37^{\circ} \mathrm{C}$, time during which the internalised integrins accumulate primarily in early endosomes ${ }^{32}$. The proportion of surface-labelling integrin antibody that remained on the cell surface was then quenched with an antiAlexa488 antibody on ice, as previously described ${ }^{15}$. Cells were subsequently incubated at $37^{\circ} \mathrm{C}$ and re-emergence of internalized $\beta 1$ integrins at the basal membrane was followed by live cell TIRF microscopy imaging (Fig. 1e). In the non-malignant human mammary epithelial cell-line MCF10A, about $70 \%$ of the chased integrin was detected at the plasma membrane already after 10 minutes (Fig 1f,g). Depletion of ErbB3 by RNAi interference using two independent siRNAs reduced recycling of $\beta 1$ integrins by $40-50 \%$ (Fig. 1f,g and S1a). Similar results were obtained on primary human breast epithelial cells prHMECs (Fig. 1h,i,S1a) . The initial amount of fluorophore-labelled integrin $\beta 1$ detected on the cell surface, prior to tracing, was 18-19\% lower upon silencing of ErbB3 compared with control in MCF10A (Fig. S1c,d) but unchanged in prHMECs (Fig. S1e,f) as determined by confocal imaging. No significant change in integrin $\beta 1$ mRNA levels, as determined by quantitative RT-PCR, was observed upon depletion of ErbB3 (Fig. S1 h).

Since ErbB3 is kinase-inactive it is thought to act in a dimer with other members of the ErbB family. In order to determine if either EGFR or ErbB2 plays a similar role to ErbB3 in endocytic recycling of $\beta 1$ integrins, we subjected MCF10A cells to RNAi-mediated depletion of EGFR or ErbB2 prior to assaying endocytic recycling of integrin $\beta 1$. ErbB4 was excluded from the experiement since is not expressed in these cells ${ }^{33,34}$. Depletion of EGFR or ErbB2 did not impair integrin $\beta 1$ recycling (Fig. 1j-k, S1m), suggesting that ErbB3 acts independently of its canonical heterodimer partners to promote endocytic recycling of $\beta 1$ integrins. Efficiency of RNAi-depletion was validated by immunoblotting (Fig. S2b).

Ectopic expression of siRNA-resistant ErbB3 restored the surface pool of traced surfacelabelled $\beta 1$ integrin in ErbB3-depleted MCF10 cells, as visualized by confocal imaging (Fig. S2c,d), indicating that off-target effects of the ErbB3 siRNAs do not underlie the observed recycling defect.

Notably, the recycling assays (Fig. 1, S1) were conducted in cell culture medium devoid of growth factors. Under these conditions we did not detect tyrosine phosphorylation of immunoprecipitated ErbB3 by immunoblotting with an antibody that recognises global phosphotyrosine, nor did we detect phosphorylation of ErbB3 on Tyr1289 which is a known EGFR or ErbB2 phosphorylation site (Fig. S2a), or significant activation of the Akt and Erk1/2 kinases, typically observed downstream of ligand-stimulated ErbB3 (Fig. S2b). These results indicate that ErbB3 promotes integrin recycling in a manner that does not require ligand-induced receptor signalling. 


\section{ErbB3 promotes delivery of $\beta 1$ integrins to the leading edge of motile epithelial cell sheets}

Endocytic recycling is important for polarised distribution of integrins in migrating cells ${ }^{32-36}$ and integrin $\beta 1$ localizes to the leading edge of migrating epithelial cells in a Rab4-dependent manner ${ }^{15,17}$. To test if ErbB3 regulates the distribution of recycled integrin $\beta 1$ in sheets of migrating epithelia, a scratch was inflicted on confluent monolayers of MCF10A cells and incubated with surface labelling Alexa488-conjugated integrin $\beta 1$ antibody for 1 hour on ice, prior to removal of the antibody and subsequent incubation at $37^{\circ} \mathrm{C}$ for 1 hour (Fig. 2a). Chased integrin $\beta 1$ and F-actin was visualised in cells at the leading front of the closing cell-sheets by immunofluorescence imaging (Fig. $2 b)$. Enrichment of $\beta 1$ integrins at the leading edge, or along cell-cell contacts relative to adjacent cytoplasm was quantified in cells bordering the scratch (boxed regions in Fig 2b). RNAi-mediated depletion of ErbB3 led to a 59.6\% ( $\mathrm{p}=0.0016)$ decrease in integrin $\beta 1$ enrichment at that leading edge, as compared with control siRNA treated cells after 1 hour of tracing at $37^{\circ} \mathrm{C}$ (Fig. 2c). At cell-cell contacts, integrin $\beta 1$ enrichment was decreases with $42.5 \%$ ( $\mathrm{p}<0.0001)$ (Fig. 2d). These results are consistent with a role of ErbB3 in recycling $\beta 1$ integrins to the leading edge of migrating cells and to a lesser extent cell-cell contacts.

Given that endocytic recycling of $\beta 1$ integrins is important for cell motility one would predict that loss of ErbB3 impairs cell migration. We therefore subjected control or ErbB3 siRNA-transfected MCF10A cells to a scratch "wound healing" assay. siRNA-depletion of ErbB3 reduced the rate of scratch closure compared to control cells (Fig. 2e,f), with area under curve increasing with $50.2 \%$ and $48.2 \%$ in mock vs. lapatinib-treated samples respectively (Fig. $2 \mathrm{~g}$ ). The assay was performed in EGFdeprived but serum containing media in the presence or absence of the dual EGFR/ErbB2 specificity inhibitor lapatinib, in order to eliminate influence of canonical EGF-induced cell migration ${ }^{40,41}$. Cell proliferation as determined by EdU incorporation assays did not differ between control or ErbB3 siRNA-transfected cells (Fig. 2h), and thus impaired proliferation does not contribute to the observed migration defect caused by loss of ErbB3. In summary, our data show that ErbB3 promotes migration of epithelial sheets in a manner that does not require canonical transphosphorylation of ErbB3 by EGFR or ErbB2.

\section{ErbB3 promotes recycling of the transferrin receptor but not transport of VSV-G-ts-GFP from} the trans-golgi-network to the plasma membrane.

To address if ErbB3 regulates endocytic recycling in a cargo-specific or unspecific manner we explored if depletion of ErbB3 also influences trafficking of the transferrin receptor (TfR). The TfR has been extensively studied as a prototype cell surface receptor that undergoes continuous internalisation and effective recycling with negligable sorting to late endosomes and lysosomes ${ }^{43}$. 
Cell surface TfR on MCF7 cells was tagged with Alexa594-conjugated transferrin on ice for 30 minutes. Unbound labelled transferrin was washed away and cells incubated at $37^{\circ} \mathrm{C}$ in the presence of unlabelled transferrin for up to an hour. Cells were brought back on ice and subjected to low $\mathrm{pH}$ treatment to quench Alexa594 that had returned to the cell surface, followed by visualisation of chased transferrin retained in endosomes by fluorescence imaging (Fig. 3a). After 1 hour at $37^{\circ} \mathrm{C}$, most TfR had recycled back to the plasma membrane as indicated by the shedding of labelled transferrin from the cells. By contrast, half of the chased transferrin was still retained in endosomes in cells treated with ErbB3 siRNA (Fig. 3a,b). TfR recycles via both Rab4 and Rab11-positive compartments $^{42,43}$. Our data shows a marked reduction in TfR recycling in ErbB3-depleted cells already 10 minutes post onset of tracing, which corresponds to the time when co-localisation of Rab4 with traced TfR has been reported to peak, preceding Rab11 and TfR co-localisation that peaks at about 30 minutes $^{43}$. Our result is therefore consistent with ErbB3 promoting Rab4-dependent recycling of TfR, although it does not exclude its involvement in Rab11-dependent recycling.

In order to address if ErbB3 specifically regulates the recycling pathway or if it plays a more general role in exocytic trafficking, we utilised a well-established system to analyse exocytic trafficking using ectopically expressed vesicular stomatitis virus, VSV-G-ts-GFP. This temperature sensitive form of VSV-G accumulates in the endoplasmic reticulum (ER) at $40^{\circ} \mathrm{C}$. Upon shift to $32^{\circ} \mathrm{C}$ the protein traffics via the TGN to the cell surface (Fig. 3c). We found that silencing of ErbB3 did not affect the amount of VSV-G that was transported from the ER to the cell surface after 30 minutes, 1 hour (Fig. 3d,e) or 2 hours (Fig. S3a) at permissive temperature as monitored by immunoblotting of isolated surface-biotinylated proteins. Immunoblotting against the transmembrane protein Muc1 on the isolated biotinylated proteins control for equal sampling. To complement the surface-biotinylation assay, traced VSV-G-ts-GFP was visualised by confocal imaging. In both control or ErbB3 siRNAtransfected cells VSV-G-ts-GFP exhibited predominant localisation in the ER at $40^{\circ} \mathrm{C}$. Following shift to $32^{\circ} \mathrm{C}$, VSV-G-ts-GFP was first detected in GM130+ trans golgi network at 30 min and E-cadherin+ membrane compartment at $1 \mathrm{~h}$ in both control and ErbB3 siRNA transfected cells. (Fig. S3b).

Taken together, these results suggest a role for ErbB3 in promoting endocytic recycling of different cell surface derived receptors ( $\beta 1$ integrins and TfR), but not a general role in exocytic trafficking.

\section{Loss of ErbB3 promotes lysosomal degradation of internalised $\beta 1$ integrins}

We next set out to investigate the fate of internalised integrin $\beta 1$ that fails to recycle back to the cell surface in the absence of ErbB3. MCF10A cells were labelled with an Alexa488-conjugated surface-binding integrin $\beta 1$ antibody on ice, followed by incubation at $37^{\circ} \mathrm{C}$ for 15 minutes to allow integrin $\beta 1$ internalisation and subsequent quenching on ice of the integrin $\beta 1$ that remained on the cell 
surface. Finally, the cells were returned to $37^{\circ} \mathrm{C}$ and the fate of the internalised pool of integrin $\beta 1$ visualised by confocal fluorescence microscopy for up to half an hour (Fig. 4a,b). Already after 15 minutes of tracing, integrin $\beta 1$ levels decreased by $42.4 \%$ in ErbB3-depleted cells at which time no significant change was observed in control siRNA transfected cells (Fig. 4b,c). Notably, integrin $\beta 1$ levels were somewhat reduced already at onset of the tracing $(0 \mathrm{~h}$ time point $)$ in ErbB3-depleted cells compared to control cells (13.3\%) (Fig. 4c), which is probably due to accelerated integrin $\beta 1$ turnover in ErbB3 depleted cells evident within the 15 minute internalisation step that precedes onset of tracing (0 min).

To assess the impact of ErbB3 on integrin $\beta 1$ turnover over an extended time period (up to 8 hours), we subjected control or ErbB3 siRNA-transfected cells to treatment with the protein synthesis inhibitor cycloheximide and monitored integrin $\beta 1$ levels by western blotting (Fig. S4a,b). While integrin $\beta 1$ protein levels did not decline significantly in control cells for up to 8 hours with blocked protein synthesis, integrin $\beta 1$ levels decreased by $72 \%(\mathrm{p}=0.047)$ in ErbB3-depleted cells after 8 hours of treatment (Fig. S4a,b). To more directly assess integrin $\beta 1$ stability, we performed a pulse-chase analysis of integrin $\beta 1$ turnover. Control or ErbB3 siRNA-transfected MCF10A cells were labelled with ${ }^{35} \mathrm{~S}$-methionine/cysteine for 1 hour, followed by chasing with un-labelled amino acids at $37^{\circ} \mathrm{C}$. Integrin $\beta 1$ was subsequently immunoprecipitated and the retained ${ }^{35} \mathrm{~S}$-label determined by radiography. The data show that cells transfected with two independent siRNAs against ErbB3 exhibited accelerated integrin $\beta 1$ turnover $(49 \%(\mathrm{p}=0.001)$ or $84 \%(\mathrm{p}=0.012)$ decrease), compared with control-treated cells (Fig. 4d,e).

The turnover of $\beta 1$ integrins is slow yet limited degradation of $\beta 1$ integrins has been found to occur in the lysosomes ${ }^{44}$. In order to determine if loss of ErbB3 promotes degradation of $\beta 1$ integrins by the lysosomes, we next traced surface-labelled integrin $\beta 1$ in control or ErbB3 siRNA-transfected MCF10A cells by confocal fluorescence microscopy, in the presence or absence of the lysosomal inhibitor chloroquine (Fig. 4f,g). Chloroquine treatment caused traced integrin $\beta 1$ to accumulate in cells bordering the migratory front of scratched monolayer of both wild type and ErbB3-depleted cells. Quantification of fluorescence intensity showed that while the level of traced integrin $\beta 1$ was reduced in the ErbB3-depleted cells relative to control siRNA transfected cells (by 44.8\%, p<0.0001), consistent with its increased turnover, chloroquine treatment partially restored traced integrin $\beta 1$ levels (19.4\%, p $<0.0001$ reduction), (Fig. 4h). This result indicates that elevated integrin $\beta 1$ turnover caused by ErbB3-loss is at least in part due to increased lysosomal degradation. 


\section{ErbB3 co-localises with Rab4-positive recycling endosomes}

To test if ErbB3 resides in recycling compartments, ErbB3-mCherry was co-expressed with either GFP-Rab4 or GFP-Rab11 in MCF7 cells, followed by treatment with either primaquine, an inhibitor of endocytic recycling, or vehicle alone for 10 minutes and subsequent visualisation of the proteins by confocal imaging (Fig. 5a). Co-localisation was analysed as enrichment of ErbB3 fluorescence in Rab4 or Rab11-positive compartments (Fig. 5b) or visualised as average intensity projections of all structures (Fig. 5c). ErbB3-mCherry was not significantly enriched in GFP-Rab4 or GFP-Rab11 positive structures in the absence of primaquine-treatment. However, upon primaquine-treatment for 10 minutes, enrichment of ErbB3 in Rab4-positive structures increased 6.2 fold ( $\mathrm{p}=0.0003$ ), while no significant enrichment was observed in Rab11-positive structures (Fig. 5b). The preferred ErbB3 colocalisation with Rab4-positive rather than Rab11-positive recycling endosomes, is consistent with a role for ErbB3 in promoting Rab4-dependent recycling. This line with with the observation that ErbB3 is required for endocytic recycling of integrin $\beta 1$ already within 10 minutes after internalization (Fig. 1e-i), during which time the rapid Rab4-dependent recycling route is prominent ${ }^{12,13}$. Notably, our data does however not exclude the possibility that ErbB3 influences alternative recycling routes dependent on other Rab proteins.

\section{ErbB3 promotes assembly of the Arf6-Rabaptin5-GGA3 sorting complex and stability of Rabaptin5 and GGA3}

We next explored whether or not ErbB3 physically interacts with $\beta 1$ integrins or selected components of the machinery implicated in sorting $\beta 1$ integrins for Rab4-dependent fast recycling. Towards this end, we immunoprecipitated endogenous ErbB3 from MCF10A cell extracts and examined co-precipitates by western blotting against selected candidates. We found that ErbB3 coprecipitated efficiently with both the Arf GTPase-binding protein GGA3 and the Rab4-binding Rab5effector Rabaptin5 at endogenous levels (Fig. 6a). The interaction of ErbB3 with GGA3 or Rabaptin5 increased after a 30-minute treatment with primaquine, consistent with them interacting in the recycling compartment. GGA3 has previously been shown to be required for Rab4-dependent recycling of $\beta 1$ integrins and c-Met ${ }^{15,16}$, while Rabaptin5, which is an accessory protein of GGA family members ${ }^{45}$, has been shown to regulate Rab4-dependent recycling of $\beta 3$ integrins ${ }^{28}$. GGA3 and Rabaptin5 are therefore plausible effectors of ErbB3 in the control of endocytic recycling.

GGA3 has been shown to interact with Arf6 to drive recycling of different transmembrane proteins ${ }^{17,46}$. We therefore set out to determine if loss of ErbB3 might influence formation of a putative Arf6-GGA3-Rabaptin5 complex. MCF10A cells were transfected with control or ErbB3 siRNA, prior to treatment with primaquine or vehicle alone for 10 minutes. Harvested cell lysates were 
subsequently subjected to immunoprecipitation of Arf6 and western blotting for bound GGA3 and Rabaptin5 (Fig. 6b). The result showed that loss of ErbB3 indeed reduced the amount of GGA3 and Rabaptin5 that associated with Arf6 both in the absence and in particular in the presence of primaquine which interestingly is when we observe a stronger co-immunoprecipitation of ErbB3 with GGA3 and Rabaptin5 (Fig. 6a). However, both Rabaptin5 and GGA3 protein levels were also reduced in ErbB3-depleted cells, as estimated by intensity of western blot bands (Fig. 6c, upper panels), which may contribute to the reduced co-immunoprecipitation. Reduced protein levels of GGA3 and Rabaptin5 did not correlate with a comparable reduction in gene transcription, as determined by RTPCR (Fig. 6c, lower panels), while treatment of ErbB3-depleted cells with the proteasome-inhibitor MG231 partially restored GGA3 and Rabaptin5 protein levels (Fig. S5a). These data indicate that in absence of ErbB3, GGA3 and Rabaptin5 are destabilised, at least in part through proteasomal degradation, presumably as a consequence of their reduced engagement in a stable complex with Arf6 scaffolded by ErbB3.

Interestingly, we noted that ErbB3 has a putative GGA3-binding motif (DxxLL) exposed on the N-lobe of the ErbB3 kinase domain (Fig. 6d), similar to what has been found on TrkA RTK ${ }^{47}$. The DxxLL motif constitutes a consensus binding site for VHS domains found in GGA proteins. To test if ErbB3 binds GGA3 via this motif, we mutated the two conserved leucines in ErbB3 to alanine (LL866/7AA). GGA3 was overexpressed with or without ErbB3 wildtype or the LL866/7AA mutant in HEK293T cells followed by immunoprecipitation of ErbB3 and western blotting against GGA3 (Fig. 6e). We found that GGA3 co-immunoprecipitated with wild-type but not the LL866/7AA mutant ErbB3 (Fig. 6e) suggesting that ErbB3 interacts with the VHS domain of GGA3 (Fig. 6d). We next tested the hypothesis that ErbB3 scaffolds the assembly of the Arf6-GGA3-Rabaptin5 complex by ectopically expressing these proteins in HEK293T cells with or without wild-type or LL866/7AA mutant ErbB3, followed by immunoprecipitation of Arf6 and western blot analysis of associated ErbB3, GGA3 and Rabaptin5. We found that GGA3 and Rabaptin5 co-precipitated more efficiently with Arf6 in the presence of wild-type but not mutant ErbB3(LL866/7AA) (Fig. 6f). These results suggest that ErbB3 promotes assembly of an Arf6-GGA3-Rabaptin5 endosomal sorting complex. Apart from interacting with GGA3, and in line with a scaffolding function for ErbB3, we found that ErbB3 directly binds Rabaptin5 in vitro, in pull-down experiments of recombinant GST-ErbB3 incubated with recombinant MBP-Rabaptin5 (Fig. S5b).

Taken together, our data suggest that ErbB3 acts as a scaffold that associates with both GGA3 and Rabaptin5 to promote the assembly of the Arf6-GGA3-Rabaptin5 endosomal sorting complex. In the absence of ErbB3, GGA3 and Rabaptin5 protein levels are reduced presumably as a consequence of their release from the sorting complex. 


\section{Rabaptin5 regulates recycling of $\beta 1$ integrins}

Previous studies reveal critical importance of Rabaptin5 for directing $\beta 3$ integrins towards Rab4-dependent recycling ${ }^{28,48}$, however the same has not yet been demonstrated for $\beta 1$ integrins. We therefore traced surface-labelled $\beta 1$ integrins in MCF10A cells transfected with either control or Rabaptin5 siRNA and visualised the re-emergence of internalized $\beta 1$ integrins at the basal membrane by TIRF live cell imaging (as outlined in Fig. 7a). Depletion of Rabaptin5 caused a reduction in the rate and amount of $\beta 1$ integrins that recycled back to the basal membrane (Fig. 7b,c), quantified as a $28.7 \%(\mathrm{p}=0.041)$ reduction in area under curves (AUC) (Fig. 7d).

Furthermore, we examined the impact of Rabaptin5 depletion on integrin $\beta 1$ stability by pulse-chase analysis of protein turnover. Control or Rabaptin5 siRNA-transfected MCF10A cells were labelled with ${ }^{35} \mathrm{~S}$-methionine/cysteine for 1 hour, followed by chasing with un-labelled amino acids at $37^{\circ} \mathrm{C}$. Integrin $\beta 1$ was subsequently immunoprecipitated and retained ${ }^{35} \mathrm{~S}$-label was determined by autoradiography. The data shows that Rabaptin5-depleted cells exhibited accelerated integrin $\beta 1$ turnover, $49 \%$ ( $\mathrm{p}=0.014)$ reduction in 10 hours as compared with control-treated cells (Fig. 7e,f). Similarly, previous work has shown that siRNA-mediated silencing of GGA3 re-routes $\beta 1$ integrins from Rab4-dependent recycling towards lysosomal degradation ${ }^{17}$. Thus, loss of either Rabaptin5 or GGA3 mimics the effect of loss of ErbB3 on endocytic trafficking of $\beta 1$ integrins, consistent with the hypothesis that GGA3 and Rabaptin5 are effectors of ErbB3 in endosomal trafficking.

\section{Discussion}

In this paper we report that the RTK ErbB3 acts in a ligand-independent manner to promote endocytic recycling of both $\beta 1$ integrins and the transferrin receptor. Endocytic trafficking of RTKs provides critical spatial and temporal control of the intracellular signalling events they trigger, which ultimately governs cellular response. Hence, the mechanisms by which RTKs internalise and traffic within the cell has been subject to intense scrutiny. While prevailing knowledge centers on RTKs as vesicular passengers, our study provides a compelling example of an RTK playing an integral role in the endocytic trafficking machinery per se.

RTK signalling is integrated at many levels with the regulation of endocytic trafficking of integrins $^{49-51}$. Growth factor mediated activation of Akt or protein kinase D (PKD) can for example regulate recycling of integrins by phosphorylating proteins, such as ACAP1, an ARF6GAP ${ }^{52}$, or Rabaptin $5^{28}$, respectively. Conversely, several studies have shown that integrins alter recycling of other receptors, such as $\mathrm{EGFR}^{53}$ and vascular endothelial growth factor receptor (VEGFR2) ${ }^{54}$, further highlighting the close coordination of trafficking of integrins and RTKs. We find that ErbB3 can 
promote endocytic recycling of $\beta 1$ integrins and TfR, independently of canonical ErbB3-driven tyrosine kinase signalling. Intriguingly, EGFR has been reported to function in a ligand-independent manner to scaffold an exocyst subcomplex needed to assemble autophagosomes ${ }^{55}$. This invites wider speculation that 'inactive' RTKs, as exemplified by EGFR and ErbB3, might play an instrumental role in intracellular vesicular trafficking.

We show that ErbB3 interacts with both GGA3 (via its VHS domain) and Rabaptin5 to promote assembly and stability of the Arf6-GGA3-Rabaptin5 endosomal sorting complex. GGA3 has previously been reported to be required to sort multiple cargos, such as $\beta 1$ integrins, $c-M e t$ and c-Ret for Rab4-dependent recycling ${ }^{16,17,20}$. It is therefore possible that ErbB3 drives recycling of not only $\beta 1$ integrins and TfR as reported here, but all cargo that depends on Arf6-GGA3, although this remains to be addressed. Furthermore, loss of ErbB3 redirects $\beta 1$ integrins towards lysosomes for degradation, mimicking loss of GGA3 that similarly redirects both $\beta 1$ integrin $^{17}$ and c-Met towards lysosomal degradation $^{16}$, or Rabaptin5 depletion that we find similarly causes trafficking of internalised $\beta 1$ integrin towards lysosomal degradation. Taken together these results lead us to propose that ErbB3 promotes endocytic recycling by promoting formation and stability of the Arf6-GGA3-Rabaptin5 vesicular adaptor complex.

Activation of EGFR and ErbB2 involve formation of asymmetric kinase domain dimers, whereby one kinase domain acts as an 'activator' and the other as a 'receiver', that becomes activated $^{56,57}$. However, the ErbB3 pseudo-kinase domain acts only as an activator while the receiver interface diverges in sequence from other ErbB receptor family members and is not engaged in kinase domain dimerisation ${ }^{56}$. Interestingly, GGA3 interacts with a DxxLL motif situated within the dysfunctional reciever interface in the ErbB3 pseudokinase domain. This motif is not present in other ErbB receptor family members, where the corresponding interface is instead engaged in asymmetric dimer formation. It is tempting to speculate that the dysfunctional dimer interface in ErbB3 may have evolved a novel function in regulating endocytic trafficking, shedding some light on the enigmatic nature of pseudokinases.

Previous studies have found that $\beta 1$ integrins can physically interact with EGFR and endocytose together ${ }^{53,58}$. Although ErbB3 partly co-localised with $\beta 1$ integrins at focal adhesions and cell-cell contacts we did not find that depletion of ErbB3 influenced internalisation of $\beta 1$ integrins, and it remains to be addressed if ErbB3 and $\beta 1$ integrins associate during endocytosis. Our results suggest that ErbB3 encounters $\beta 1$ integrins in sorting endosomes, presumably at the step when the cargo is sorted into Rab4-positive vesicles through engagement of the Arf6-GGA3-Rabaptin5complex. This is suggested by the observation that Integrin $\beta 1$ and ErbB3 co-localisation was primarily observed upon treatment with the recycling inhibitor primaquine, when ErbB3 is retained in a Rab4-positive compartment, which coincides with enhanced binding of ErbB3 to GGA3 and 
Rabaptin5. This is in line with previous work showing that GGA3 is enriched in Rab4-positive endosomes along with endocytosed Met, and to a much lesser extent Rab5-positive early endosomes.

The proposed role of ErbB3 in sorting of $\beta 1$ integrins and other cargo into recycling endosomes, postulates that ErbB3 frequently resides in the sorting compartment independent of ligand-stimulation. In line with this prediction, ErbB3 has been found to continuously endocytose and recycle back to the plasma membrane ${ }^{30,31,59}$ independent of both ligand stimulation and other members of the EGFR family ${ }^{30}$. Intriguingly, dynamic availability of ErbB3 in vesicular compartments may also be regulated independently of EGFR and ErbB2 since steady-state protein levels of ErbB3, but not EGFR or ErbB2, is tightly controlled by ligand-independent ubiquitination by the E3-ligase Nrdp1 that localises in perinuclear membranes and target ErbB3 for proteasomal degradation ${ }^{60,61}$.

We found that ErbB3-dependent cell sheet migration also does not require EGF or other growth factors. It is however important to stress that growth factor-induced EGFR or ErbB2 signaling is well known to promote cell migration, and our data does not rule out or address if ErbB3 cooperates with EGFR or ErbB2 to drive growth factor-induced cell motility. In this manuscript we focus entirely on the unexpected growth factor-independent role of ErbB3, leaving the more studied and complex growth factor-induced cell signaling events aside.

Our data shows that ErbB3 regulates delivery of $\beta 1$ integrins to the leading edge of migrating sheets of epithelial cells and promotes their migration. Several studies have established that recycling of integrins promotes cell migration and cancer cell invasiveness ${ }^{62,63}$. It will be interesting to investigate if ErbB3, that is frequently overexpressed in metastatic cancers, could contribute to cancer dissemination in part by promoting integrin recycling.

\section{Methods}

Cell lines and cell culture. MCF10A cells were cultured in DMEM/F12 (Gibco) supplemented with glutamine (Sigma-Aldrich), 5\% horse serum (Gibco), $20 \mathrm{ng} / \mathrm{ml}$ epidermal growth factor (EGF) (Miltenyi Biotech), $0.5 \mathrm{mg} / \mathrm{ml}$ hydrocortisone, $100 \mathrm{ng} \mathrm{ml}^{-1}$ cholera toxin and $10 \mu \mathrm{g} / \mathrm{ml}$ insulin (all from Sigma-Aldrich). Where indicated, a starvation medium lacking serum, insulin and EGF was used. Primary human mammary epithelial cells (prHMECs) from Gibco were cultured in HMEC ready medium (Invitrogen). MCF7 cells were cultured in EMEM supplemented with $0.01 \mathrm{mg} / \mathrm{ml}$ human recombinant insulin and 10\% fetal bovine serum. HEK293T cells were cultured in DMEM (SigmaAldrich) with 10\% FBS (Gibco). Unless otherwise stated cell lines were purchased from ATCC. Culture conditions were $5 \% \mathrm{CO} 2$ at $37^{\circ} \mathrm{C}$. For immunocytochemistry coverslips were coated with type I bovine collagen solution (Advanced BioMatrix) in 1:1000 in $0.02 \mathrm{M}$ acetic acid overnight prior to application of cells.

Antibodies. The following primary antibodies were used: anti-ErbB3 (clone 2F12; Upstate Cell Signaling Solutions) for IP; anti-ErbB3 (clone D22C5; Cell Signaling) for western blotting; anti- 
integrin $\beta 1$ (monoclonal, ab52971 from Abcam); anti-Rabaptin5 (monoclonal, sc-271069 from Santa Cruz Biotechnology); anti-GGA3 (clone 8; BD Transduction Laboratories); anti-Arf6 (clone 3A-1, Santa Cruz Biotech.); anti-phospho-ErbB3 Tyr1289 (\#4791, Cell Signalling Technology); anti-EGFR (\#2232, Cell Signalling Technology); anti-ErbB2 (06-562, Millipore); anti-phospho-AKT Thr308 (\#2965, Cell Signalling Technology); anti-AKT (\#9272, Cell Signalling Technology); anti-phospho ERK1/2 (\#9101, Cell Signalling Technology); anti-ERK1/2 (\#9102. Cell Signalling Technology); anti-Total p-Tyr ab (4G10 platinum); anti-E-cadherin (\#610182, BD Transduction Laboratories); antiMuc1 (Cell Signalling Technology); anti-GM130 (clone 35, BD Biosciences); anti-GAPDH (clone 14C10; Cell Signaling); anti- $\alpha$-tubulin (clone T5168, Sigma-Aldrich). For western blotting HRPconjugated goat anti-mouse or goat anti-rabbit (The Jackson Laboratory) secondary antibodies were used. Alexa fluor ${ }^{\circledR} 594$ or Alexa fluor ${ }^{\circledR} 488$-conjugated anti-rabbit antibodies (The Jackson Laboratory) were used for immunocytochemistry. For the integrin recycling assay, Alexa Fluor ${ }^{\circledR} 488$ anti-human integrin $\beta 1$ antibody (clone TS2/16, Biolegend) was used.

SiRNA transfection. Cells were transfected with RNAiMAX (Invitrogen) according to supplier's instructions. A final concentration of $20 \mathrm{nM}$ of siRNA was used. The following siRNAs were used: ErbB3\#1 siRNA (5'CAAUACCAGACACUGUACAAGCUCU53') and ErbB3\#2 siRNA (5'UCGUCAUGUUGAACUAUAA3') from Invitrogen), rabaptin5 siRNA (5'CCGGGCAAUUCUGAAUGAUACUAAA3' from Invitrogen) and scramble siRNA (nontargeting pre-designed ID 12935112, also from Invitrogen). The silencing experiments were performed $48 \mathrm{~h}$ post transfection.

Drugs and inhibitors. The EGFR/ErbB2 inhibitor lapatinib was used at $1 \mu \mathrm{M}$ (LC Laboratories) and was batch-tested for efficiency; cycloheximide at $50 \mu \mathrm{M}$ (from Sigma-Aldrich), chloroquine at $50 \mu \mathrm{M}$ (\#14774 from Cell Signaling). In all three cases, control cells were treated with vehicle alone (DMSO, from Sigma-Aldrich) at the corresponding concentration. Primaquine was used at $0.5 \mathrm{mM}$ (\#160393

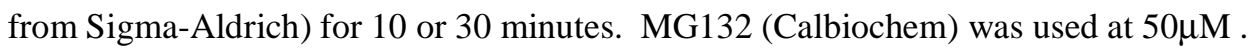

cDNAs and DNA transfection. MCF10A cells were transfected with Lipofectamine 3000 (Invitrogen) according to supplier's instructions. Constructs for Rab4, Rab11 and Rabaptin5 were kindly provided from Marino Zerial (MPG Dresden, Germany), and mCherry- and Citrine-tagged ErbB3 were kindly provided by Martin Offterdinger (Innsbruck, Austria). Rabaptin5 was subcloned into pMALC2 for bacterial expression (see below). mCherry-tagged Rabaptin5 and YFP-tagged Arf6 were from Addgene. Flag-ErbB3 cDNA was subjected to site-directed mutagenesis (Agilent) to generate LL866/7AA mutant Flag-ErbB3. For resue experiments mCherry-ErbB3 carrying two nonsense point mutations in the siRNA-recognision sequence was subcloned into pAdEasy XL and adenovirus produced using the AdEasy XL adenovirus system (Agilent) according to manifacturers 
instructions and MCF10A cells infected at a titer that yielded ErbB3 overexpression in approximately $90 \%$ of the cells.

qPCR analysis. RNA was extracted with RNeasy Mini Kit (\#74104 from Qiagen). cDNA was synthesized with iScript ${ }^{\mathrm{TM}}$ cDNA Synthesis Kit (\#170-8891 from Bio-Rad). qPCR was performed using KAPA SYBR ${ }^{\circledR}$ Fast and primers for ITGB1 (5'AGATCCGAAGTTTCAAGGGC3' and 5'GAACCATGACCTCGTTGTTC3'), GGA3 (5'GGGACAGGGTGTCTGAGAAAG3' and 5'GTGCCTCGTCTTCCTTCACC3'), RABEP1 (5'TTCCCAGCCTGACGTTTCTC3' and 5'GCTGCTGTTGTGCACGTAAA3') and GAPDH (5'CCCTTCATTGACCTCAACTA3' and 5'CCAAAGTTGTCATGGATGAC3'). The comparative Ct method was used to calculate fold change in gene expression in respect to GAPDH.

Western blotting. Cells were lysed in sodium dodecyl sulphate (SDS)-sample buffer (120 mM Tris$\mathrm{HCl} \mathrm{pH}$ 6.8, $3 \%$ SDS, $15 \%$ glycerol, $0.03 \%$ bromophenol blue, $75 \mathrm{mM}$ dithiothreitol (DTT) and run on $8-10 \%$ polyacrylamide gels. Proteins were transferred onto Hybond ${ }^{\mathrm{TM}}-\mathrm{P}$ polyvinylidene fluoride (PVDF) membranes at $100 \mathrm{~V}$ for $120 \mathrm{~min}$ at $4{ }^{\circ} \mathrm{C}$. Membranes were blocked in $4 \%$ milk or $5 \%$ bovine serum albumin (BSA), dissolved in TTBS (tris-buffered saline, $0.1 \%$ Tween 20). The protein bands were visualised using ECL ${ }^{\mathrm{TM}}$ or ECL plus ${ }^{\mathrm{TM}}$ reagents and Hyperfilm ${ }^{\circledR}$ ECL (all from GE Healthcare) and detected with a CCD camera (Biorad). Quantification of western blots was performed with ImageJ software.

Immunoprecipitation. For ErbB3 immunoprecipitation cells were lysed in: 1\% NP40, 50mM Tris $\mathrm{HCl} \mathrm{pH} 8.0,150 \mathrm{mM} \mathrm{NaCl}, 0.5 \%$ Sodium Deoxycholate, $0.1 \%$ SDS and 1mM EDTA supplemented with Halt ${ }^{\mathrm{TM}}$ phosphatase/protease inhibitor (ThermoFisher). Preclearing of lysates was performed by incubating samples for $1 \mathrm{~h}$ at $4^{\circ} \mathrm{C}$ under rotation with Pierce control agarose resin. Antibody incubation was performed overnight $4{ }^{\circ} \mathrm{C}$ under rotation and precipitates washed 4 times in lysis buffers. Precipitated proteins were resolved by western blotting. Arf6 immunoprecipitations was performed as above but with lysis buffer: $1 \%$ Triton-X100, $100 \mathrm{mM}$ sodium chloride $(\mathrm{NaCl}), 50 \mathrm{mM}$ Tris-HCl pH 7.4.

Immunocytochemistry. MCF10A or MCF7 cells were cultured on collagen I-coated coverslips and fixed with 4\% PFA in PBS for 20 min, permeabilized with 0.5\% Triton in PBS for $10 \mathrm{~min}$ and then blocked in Superblock T20 blocking buffer (Thermo Scientific). Primary and secondary antibodies and, where indicated, Alexa fluor ${ }^{\circledR}$ 488-conjugated Phalloidin (Life Technologies) were diluted in blocking buffer. Coverslips were mounted using ProLong ${ }^{\circledR}$ Antifade Reagent (Life Technologies) with or without DAPI. Images were taken with a Leica SP8 (confocal) or Zeiss Axio Imager M2 (epifluorescence) microscopes and processed using Leica Suite or Zeiss ZEN 2011 software, respectively. Deconvolution of confocal image stacks was carried out in Huygens Essential 19.04 
(Scientific Volume Imaging B.V.) using a theoretical PSF, automatic background estimation and CMLE deconvolution algorithm. The final signal to noise ratio was set to 10 .

Integrin recycling assay. MCF10A cells were plated on collagen-coated 24-well plates either sparsely or at high confluency for subsequent scratching. Cells were growth factor deprived for $3 \mathrm{~h}$ and then put on ice for 5 minutes to cool down. Surface integrin $\beta 1$ was labelled at $4{ }^{\circ} \mathrm{C}$ during 45 minutes, then washed twice with PBS and returned to $37^{\circ} \mathrm{C}$ in order to allow internalisation. After 15 minutes, cells were put back on ice and treated for $1 \mathrm{~h}$ with anti-Alexa fluor ${ }^{\circledR} 488$ (\#A11094 from Life Technologies) in order to quench remaining surface signal. After quenching, cells were again washed twice with PBS and incubated in deprivation media at $37^{\circ} \mathrm{C}$. At indicated times cells were fixed with PFA 4\% in PBS for 20 minutes at room temperature. When only integrin internalisation was monitored, the quenching step was not performed. Quantification of total pixel intensity per cell, mean pixel intensity and average projections was performed with ImageJ.

TIRF microscopy. A prism-type TIRF microscope built around a Nikon E600FN upright microscope equipped with a 16X 0.8-NA water-immersion objective was used. The output from diode-pumped solid-state lasers (Cobolt, Stockholm, Sweden) were merged by dichroic mirrors and homogenized and expanded by a rotating, light-shaping diffusor (Physical Optics Corp., Torrance, CA). Excitation light was selected by interference filters (491 nm for Alexa-488 and $561 \mathrm{~nm}$ for mRFP, Chroma) mounted in a filter wheel (Lambda 10-3, Sutter Instruments, Novato, CA) and refocused through a modified dove prism (Axicon, Minsk, Belarus) at a $70^{\circ}$ angle to achieve total internal reflection. Cells grown on 25-mm glass coverslips (Menzel-Gläser, \#1) were kept on ice and mounted in a modified Sykes-Moore perfusion chamber just before imaging, placed on top of the prism and perfused with $\mathrm{DMEM}+\mathrm{F} 12$ at a rate of $0.1 \mathrm{~mL} / \mathrm{min}$. Emission wavelengths were selected with interference filters (525/25 nm for Alexa 488, 590/20 for mRFP, Chroma) mounter in a filter wheel (Sutter Instruments) and fluorescence was detected by a back-illuminated EM-CCD camera (DU-887, Andor Technology, Belfast, Northern Ireland). Filter wheels and camera were controlled by MetaFluor (Molecular Devices Corp., Downington, PA) and images were acquired every $10 \mathrm{~s}$. An electronic shutter prevented light exposure between image captures. Imaging was done at $37^{\circ} \mathrm{C}$.

TIRF microscopy images were analysed off-line using ImageJ. Briefly, cell footprints were manually identified and regions of interest covering the edges of the adherent cells were draw. Intensity changes within these regions through the time course of the experiment (45 $\mathrm{min}$ ) were measured and exported to Excel. All data points were background corrected, followed by normalization to the pre-stimulatory level (F/F0). Cells within large cell clusters were excluded from the analysis.

Transferrin recycling assay. MCF7 cells were incubated in starvation media supplemented with 25 $\mathrm{mM}$ HEPES for $3 \mathrm{~h}$ prior to the assay. Cells were treated with $5 \mu \mathrm{g} / \mathrm{mL}$ of Alexa fluor ${ }^{\circledR}$-conjugated 
transferrin (T13343, from ThermoFisher) for 30 minutes on ice. Thereafter, excess of labelled transferrin was removed by three washes with PBS and subsequently incubated in media containing 50 $\mu \mathrm{g} / \mathrm{ml}$ unlabelled holo-transferrin (T4132, from Sigma-Aldrich) for indicated times $(0,10,30,60$ minutes). Finally, the cells were treated with an acidic solution $(0.2 \mathrm{M}$ acetic acid and $0.5 \mathrm{M} \mathrm{NaCl})$ for 30 seconds to remove surface labelling, fixed in 4\% PFA in PBS for 20 minutes at room temperature and mounted. Quantification performed on tree independent experiment. Mean cell intensity was measured with ImageJ ( $n>17$ images of 1-4 cells for each data point). MCF7 cells were chosen for this assay due to efficient surface-labeling with Alexa fluor ${ }^{\circledR}$-conjugated transferrin. Attemps to perform the transferrin-recycling assay in MCF10A cells were unsucsessful, due to insufficient surfacelabelling with Alexa fluor ${ }^{\circledR}$-conjugated transferrin in these cells.

Wound healing assay. MCF10A cells were seeded in Falcon Multiwell 48-well plates and grown to full confluency. Before performing the wound, cells were pre-treated with lapatinib $1 \mu \mathrm{M}$ or DMSO alone in serum-containing media lacking added growth factors for 1 hour. Thereafter, a scratch was placed in the middle of each well with a sterile pipette tip. After two washes with PBS, lapatinib/DMSO-containing growth-factor deprived but serum containing media was added back to the cells and the wound healing process was monitored using an IncuCyte ZOOM 40008 microscope for $14 \mathrm{~h}$. The obtained images were analysed using FIJI software.

${ }^{35} \mathrm{~S}-\mathrm{Met} / \mathrm{Cys}$ pulse-chase protein turnover assay. MCF10A cells transfected with control or ErbB3 siRNA were incubated with growth factor deprived DMEM media lacking methionine and cysteine supplemented with $10 \mu \mathrm{Ci} / \mathrm{ml}{ }^{35} \mathrm{~S}$-methionine/cysteine (Perkin Elmer) for 30 minutes at $37^{\circ} \mathrm{C}$. The labelling media was replaced with growth factor deprived MCF10A culture media containing $5 \mathrm{mM}$ L-cysteine and $5 \mathrm{mM} \mathrm{L-methionine} \mathrm{(Sigma)} \mathrm{for} \mathrm{indicated} \mathrm{times,} \mathrm{prior} \mathrm{to} \mathrm{cell} \mathrm{lysis} \mathrm{and}$ immunoprecipitation of integrin $\beta 1$, SDS-polyacrylamide (PAGE) gel electrophoresis and detection of incorporated radioactivity using a Phosphoimager. Images were scanned and quantification of three independent experiments was perform in ImageJ.

Recombinant protein purification and in vitro binding assay. pMalC2-rabaptin5 was transformed into the BL21S(DE3)pLysS bacterial strain (Invitrogen). Protein expression was induced with $0.3 \mathrm{mM}$ IPTG for 2 hours at $37^{\circ} \mathrm{C}$ and recombinant MBP-rabaptin5 was purified with an amylose resin (New England Biolabs) according to manufacturs instructions and dialysed against lysis buffer (20 $\mathrm{mM}$ Tris$\mathrm{HCl}$ ph7.4, $50 \mathrm{mM} \mathrm{NaCl}, 0.05 \mathrm{mM}$ PMSF, $6 \mathrm{KIU} / \mathrm{ml}$ aprotinin $0.5 \mathrm{mM}$ DTT, 5\% glycerol and $0.5 \%$ Triton X-100). Recombinant 6His-ErbB3 was purchased from Sigma. The in vitro binding assay was conducted by incubating $1 \mu \mathrm{g} / \mathrm{ml}$ recombinant ErbB3 with $1 \mu \mathrm{g} / \mathrm{ml}$ recombinant Rabaptin5 in a stringent buffer containing $50 \mathrm{mM}$ Tris, $\mathrm{pH} 7.5,150 \mathrm{mM} \mathrm{NaCl}, 1 \% \mathrm{NP} 40,0.5 \%$ sodium deoxycholate, $0.1 \%$ SDS, $1 \mathrm{mM}$ EDTA, HALT protease and phosphatase inhibitor overnight at $4^{\circ} \mathrm{C}$. ErbB3 was immunoprecipitated and bound Rabaptin5 detected by western blotting. 
Colocalization analysis. Analysis was conducted on confocal fluorescence images and analysed using Image J. Rab4/11 and ErbB3 colocalization: Single Rab4 or Rab11 positive structures were identified and 40x40 pixel squares were drawn with the structures in the center and saved as separate images. For each cell, a minimum of 15 structures were randomly selected and the intensity of the center (7pixel diameter circle, a) and the adjacent volume (background, b) for each structure was determined using ImageJ. These regions were subsequently transferred to the other channel (ErbB3) and similar measurements were performed. The relative enrichment of ErbB3 at the Rab-positive structures was determined by the formula (a-b)/b. An average for each cell was determined, and a minimum of 16 cells was analyzed for each condition. Images shown in the paper are average projections of all structures from all cells analyzed. Statistical analysis was performed using unpaired, 2-tailed Student's t-test. ErbB3 and integrin $\beta 1$ colocalization: Deconvolved confocal image stacks were processed into maximum intensity projections using Fiji ImageJ. Signal intensities along manually drawn lines were obtained using the histogram tool. Colocalisation was analysed in image $\mathrm{J}$ as enrichment of integrin $\beta 1$ in ErbB3 positive structures (as described for ErbB3 and Rab co-localisation above).

VSV-G trafficking assay. VSV-G ts045-GFP was expressed in MCF10A cells for 24 hours. Cells were incubated at $40^{\circ} \mathrm{C}$ for 6 hours, which causes misfolding and trapping of VSV-G in the ER. The cells were then shifted to permissive temperature $\left(32^{\circ} \mathrm{C}\right)$ and either PFA-fixed at indicated times for immunofluorescence imaging or subjected to incubation on ice with Sulfo-NHS-SS-Biotin (Thermo Scientific) to biotinylate all surface proteins. Biotinylated cells were lysed in $1 \%$ Triton X-100 containing lysis buffer followed by pulldown with Streptavidin-agarose beads (Sigma) overnight and western blot analysis with indicated antibodies.

EdU incorporation assay. EdU incorporation into DNA of MCF10A monolayers subjected to a scratch assay in presence of Lapatinib $1 \mathrm{uM}$ or vehicle alone was detected using the Click-iT EdU Alexa-488 Fluor Imaging kit (Invitrogen) following the manufacturer's instructions. Cells were later fixed, double stained with DAPI and mounted for microscope imaging with Zeiss Axio Imager M2. Quantification of EdU-positive cells at indicated times in control or ErbB3-silenced cells was performed with ImageJ. 


\section{References}

1. Schlessinger, J. Ligand-induced, receptor-mediated dimerization and activation of EGF receptor.

Cell 110, 669-672 (2002).

2. Tiwary, S. et al. ERBB3 is required for metastasis formation of melanoma cells. Oncogenesis $\mathbf{3}$, e110 (2014).

3. Pradeep, S. et al. Hematogenous metastasis of ovarian cancer: rethinking mode of spread. Cancer Cell 26, 77-91 (2014).

4. Smirnova, T. et al. Phosphoinositide 3-kinase signaling is critical for ErbB3-driven breast cancer cell motility and metastasis. Oncogene 31, 706-715 (2012).

5. Yoshioka, T. et al. Significance of integrin $\alpha v \beta 5$ and erbB3 in enhanced cell migration and liver metastasis of colon carcinomas stimulated by hepatocyte-derived heregulin. Cancer Sci. 101, 2011-2018 (2010).

6. Gaborit, N., Lindzen, M. \& Yarden, Y. Emerging anti-cancer antibodies and combination therapies targeting HER3/ERBB3. Hum. Vaccines Immunother. 12, 576-592 (2016).

7. Sorkin, A. \& Goh, L. K. Endocytosis and intracellular trafficking of ErbBs. Exp. Cell Res. 315, 683-696 (2009).

8. Avraham, R. \& Yarden, Y. Feedback regulation of EGFR signalling: decision making by early and delayed loops. Nat. Rev. Mol. Cell Biol. 12, 104-117 (2011).

9. Miaczynska, M. \& Bar-Sagi, D. Signaling endosomes: seeing is believing. Curr. Opin. Cell Biol. 22, 535-540 (2010).

10. Al-Akhrass, H. et al. Sortilin limits EGFR signaling by promoting its internalization in lung cancer. Nat. Commun. 8, 1182 (2017).

11. Wiley, H. S. Trafficking of the ErbB receptors and its influence on signaling. Exp. Cell Res. 284, $78-88$ (2003).

12. Tomas, A., Futter, C. E. \& Eden, E. R. EGF receptor trafficking: consequences for signaling and cancer. Trends Cell Biol. 24, 26-34 (2014).

13. Bridgewater, R. E., Norman, J. C. \& Caswell, P. T. Integrin trafficking at a glance. J. Cell Sci. 125, 3695-3701 (2012).

14. Sahgal, P. et al. GGA2 and RAB13 promote activity-dependent $\beta 1$-integrin recycling. J. Cell Sci. 132, (2019).

15. Arjonen, A., Alanko, J., Veltel, S. \& Ivaska, J. Distinct recycling of active and inactive $\beta 1$ integrins. Traffic Cph. Den. 13, 610-625 (2012). 
16. Parachoniak, C. A., Luo, Y., Abella, J. V., Keen, J. H. \& Park, M. GGA3 functions as a switch to promote Met receptor recycling, essential for sustained ERK and cell migration. Dev. Cell 20, 751-763 (2011).

17. Ratcliffe, C. D. H., Sahgal, P., Parachoniak, C. A., Ivaska, J. \& Park, M. Regulation of Cell Migration and $\beta 1$ Integrin Trafficking by the Endosomal Adaptor GGA3. Traffic Cph. Den. 17, 670-688 (2016).

18. Puertollano, R. \& Bonifacino, J. S. Interactions of GGA3 with the ubiquitin sorting machinery. Nat. Cell Biol. 6, 244-251 (2004).

19. Zhao, Y. \& Keen, J. H. Gyrating clathrin: highly dynamic clathrin structures involved in rapid receptor recycling. Traffic Cph. Den. 9, 2253-2264 (2008).

20. Crupi, M. J. F. et al. GGA3-mediated recycling of the RET receptor tyrosine kinase contributes to cell migration and invasion. Oncogene 39, 1361-1377 (2020).

21. Zhu, G. et al. Structural basis of Rab5-Rabaptin5 interaction in endocytosis. Nat. Struct. Mol. Biol. 11, 975-983 (2004).

22. Zhai, P. et al. The interaction of the human GGA1 GAT domain with rabaptin-5 is mediated by residues on its three-helix bundle. Biochemistry 42, 13901-13908 (2003).

23. Vitale, G. et al. Distinct Rab-binding domains mediate the interaction of Rabaptin-5 with GTPbound Rab4 and Rab5. EMBO J. 17, 1941-1951 (1998).

24. Stenmark, H., Vitale, G., Ullrich, O. \& Zerial, M. Rabaptin-5 is a direct effector of the small GTPase Rab5 in endocytic membrane fusion. Cell 83, 423-432 (1995).

25. Nagelkerken, B. et al. Rabaptin4, a novel effector of the small GTPase rab4a, is recruited to perinuclear recycling vesicles. Biochem. J. 346 Pt 3, 593-601 (2000).

26. de Renzis, S., Sönnichsen, B. \& Zerial, M. Divalent Rab effectors regulate the sub-compartmental organization and sorting of early endosomes. Nat. Cell Biol. 4, 124-133 (2002).

27. Pagano, A., Crottet, P., Prescianotto-Baschong, C. \& Spiess, M. In vitro formation of recycling vesicles from endosomes requires adaptor protein-1/clathrin and is regulated by rab4 and the connector rabaptin-5. Mol. Biol. Cell 15, 4990-5000 (2004).

28. Christoforides, C., Rainero, E., Brown, K. K., Norman, J. C. \& Toker, A. PKD controls $\alpha v \beta 3$ integrin recycling and tumor cell invasive migration through its substrate Rabaptin-5. Dev. Cell 23, 560-572 (2012).

29. Deneka, M. et al. Rabaptin-5alpha/rabaptin-4 serves as a linker between rab4 and gamma(1)adaptin in membrane recycling from endosomes. EMBO J. 22, 2645-2657 (2003).

30. Sak, M. M. et al. The oncoprotein ErbB3 is endocytosed in the absence of added ligand in a clathrin-dependent manner. Carcinogenesis 33, 1031-1039 (2012).

31. Fosdahl, A. M. et al. ErbB3 interacts with Hrs and is sorted to lysosomes for degradation. Biochim. Biophys. Acta 1864, 2241-2252 (2017). 
32. Roberts, M., Barry, S., Woods, A., van der Sluijs, P. \& Norman, J. PDGF-regulated rab4dependent recycling of alphavbeta3 integrin from early endosomes is necessary for cell adhesion and spreading. Curr. Biol. CB 11, 1392-1402 (2001).

33. Haskins, J. W., Nguyen, D. X. \& Stern, D. F. Neuregulin 1-activated ERBB4 interacts with YAP to induce Hippo pathway target genes and promote cell migration. Sci. Signal. 7, ra116-ra116 (2014).

34. Wali, V. B. et al. Convergent and Divergent Cellular Responses by ErbB4 Isoforms in Mammary Epithelial Cells. Mol. Cancer Res. 12, 1140-1155 (2014).

35. Nader, G. P. F., Ezratty, E. J. \& Gundersen, G. G. FAK, talin and PIPKI $\gamma$ regulate endocytosed integrin activation to polarize focal adhesion assembly. Nat. Cell Biol. 18, 491-503 (2016).

36. Qu, F. et al. Ankyrin-B is a PI3P effector that promotes polarized $\alpha 5 \beta 1$-integrin recycling via recruiting RabGAP1L to early endosomes. eLife 5, e20417 (2016).

37. White, D. P., Caswell, P. T. \& Norman, J. C. alpha v beta3 and alpha5beta1 integrin recycling pathways dictate downstream Rho kinase signaling to regulate persistent cell migration. J. Cell Biol. 177, 515-525 (2007).

38. Ivaska, J. et al. PKCepsilon-mediated phosphorylation of vimentin controls integrin recycling and motility. EMBO J. 24, 3834-3845 (2005).

39. Powelka, A. M. et al. Stimulation-dependent recycling of integrin beta1 regulated by ARF6 and Rab11. Traffic Cph. Den. 5, 20-36 (2004).

40. Lauand, C., Rezende-Teixeira, P., Cortez, B. A., Niero, E. L. de O. \& Machado-Santelli, G. M. Independent of ErbB1 gene copy number, EGF stimulates migration but is not associated with cell proliferation in non-small cell lung cancer. Cancer Cell Int. 13, 38 (2013).

41. Maretzky, T. et al. Migration of growth factor-stimulated epithelial and endothelial cells depends on EGFR transactivation by ADAM17. Nat. Commun. 2, 229 (2011).

42. Lindsay, A. J. et al. Rab coupling protein (RCP), a novel Rab4 and Rab11 effector protein. J. Biol. Chem. 277, 12190-12199 (2002).

43. Sönnichsen, B., De Renzis, S., Nielsen, E., Rietdorf, J. \& Zerial, M. Distinct membrane domains on endosomes in the recycling pathway visualized by multicolor imaging of Rab4, Rab5, and Rab11. J. Cell Biol. 149, 901-914 (2000).

44. De Franceschi, N., Hamidi, H., Alanko, J., Sahgal, P. \& Ivaska, J. Integrin traffic - the update. J. Cell Sci. 128, 839-852 (2015).

45. Mattera, R., Arighi, C. N., Lodge, R., Zerial, M. \& Bonifacino, J. S. Divalent interaction of the GGAs with the Rabaptin-5-Rabex-5 complex. EMBO J. 22, 78-88 (2003).

46. Parachoniak, C. A., Luo, Y., Abella, J. V., Keen, J. H. \& Park, M. GGA3 functions as a switch to promote Met receptor recycling, essential for sustained ERK and cell migration. Dev. Cell 20, 751-763 (2011). 
47. Li, X., Lavigne, P. \& Lavoie, C. GGA3 mediates TrkA endocytic recycling to promote sustained Akt phosphorylation and cell survival. Mol. Biol. Cell 26, 4412-4426 (2015).

48. Do, M. T., Chai, T. F., Casey, P. J. \& Wang, M. Isoprenylcysteine carboxylmethyltransferase function is essential for RAB4A-mediated integrin $\beta 3$ recycling, cell migration and cancer metastasis. Oncogene 36, 5757-5767 (2017).

49. Ivaska, J. \& Heino, J. Cooperation between integrins and growth factor receptors in signaling and endocytosis. Annu. Rev. Cell Dev. Biol. 27, 291-320 (2011).

50. Woods, A. J., White, D. P., Caswell, P. T. \& Norman, J. C. PKD1/PKCmu promotes alphavbeta3 integrin recycling and delivery to nascent focal adhesions. EMBO J. 23, 2531-2543 (2004).

51. Caswell, P. T., Vadrevu, S. \& Norman, J. C. Integrins: masters and slaves of endocytic transport. Nat. Rev. Mol. Cell Biol. 10, 843-853 (2009).

52. Li, J. et al. Phosphorylation of ACAP1 by Akt regulates the stimulation-dependent recycling of integrin beta1 to control cell migration. Dev. Cell 9, 663-673 (2005).

53. Caswell, P. T. et al. Rab-coupling protein coordinates recycling of alpha5beta1 integrin and EGFR1 to promote cell migration in 3D microenvironments. J. Cell Biol. 183, 143-155 (2008).

54. Reynolds, A. R. et al. Stimulation of tumor growth and angiogenesis by low concentrations of RGD-mimetic integrin inhibitors. Nat. Med. 15, 392-400 (2009).

55. Tan, X., Thapa, N., Sun, Y. \& Anderson, R. A. A kinase-independent role for EGF receptor in autophagy initiation. Cell 160, 145-160 (2015).

56. Jura, N. et al. Mechanism for activation of the EGF receptor catalytic domain by the juxtamembrane segment. Cell 137, 1293 (2009).

57. Littlefield, P. et al. Structural analysis of the EGFR/HER3 heterodimer reveals the molecular basis for activating HER3 mutations. Sci. Signal. 7, ra114-ra114 (2014).

58. Yu, X., Miyamoto, S. \& Mekada, E. Integrin alpha 2 beta 1-dependent EGF receptor activation at cell-cell contact sites. J. Cell Sci. 113 ( Pt 12), 2139-2147 (2000).

59. Cao, Z., Wu, X., Yen, L., Sweeney, C. \& Carraway, K. L. Neuregulin-induced ErbB3 downregulation is mediated by a protein stability cascade involving the E3 ubiquitin ligase Nrdp1. Mol. Cell. Biol. 27, 2180-2188 (2007).

60. Diamonti, A. J. et al. An RBCC protein implicated in maintenance of steady-state neuregulin receptor levels. Proc. Natl. Acad. Sci. U. S. A. 99, 2866-2871 (2002).

61. Qiu, X.-B. \& Goldberg, A. L. Nrdp1/FLRF is a ubiquitin ligase promoting ubiquitination and degradation of the epidermal growth factor receptor family member, ErbB3. Proc. Natl. Acad. Sci. U. S. A. 99, 14843-14848 (2002).

62. Caswell, P. T. et al. Rab-coupling protein coordinates recycling of alpha5beta1 integrin and EGFR1 to promote cell migration in 3D microenvironments. J. Cell Biol. 183, 143-155 (2008).

63. Pellinen, T. \& Ivaska, J. Integrin traffic. J. Cell Sci. 119, 3723-3731 (2006). 
bioRxiv preprint doi: https://doi.org/10.1101/575449; this version posted September 10, 2020. The copyright holder for this preprint (which was not certified by peer review) is the author/funder. All rights reserved. No reuse allowed without permission.

Integral role of ErbB3 in the endocytic recycling machinery

\section{Acknowledgments}

We thank Marino Zerial, Lukas Huber and Martin Offterdinger for kindly providing cDNA constructs.

We also thank to Carl-Henrik Heldin for valuable discussion and for critically reading the manuscript.

\section{Author contributions}

A.R.S., J.S, T.T., O.I., T.M. and I.F. conceived and designed the experiments. A.R.S, J.S, O.I. and

N.D. performed the experiments and A.R.S., J.S. O.I. N.D. and I.F. interpreted the results. A.R.S. and I.F. wrote the manuscript, with input from all other authors. 


\section{Figures}

\section{a}

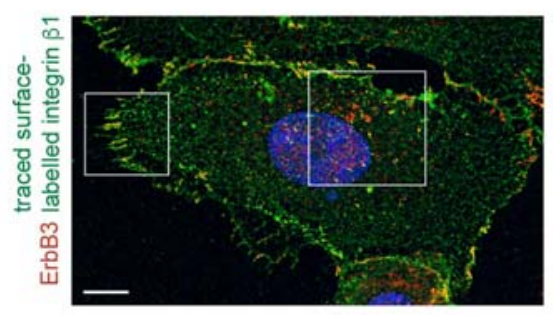

C

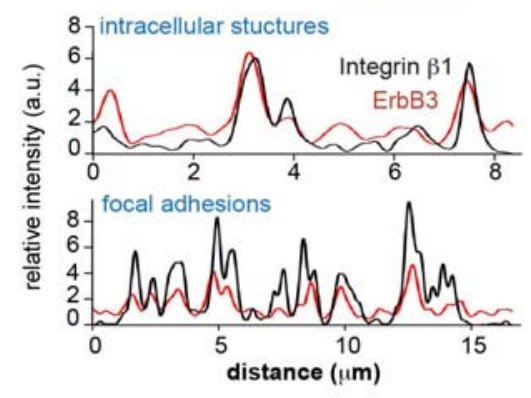

b

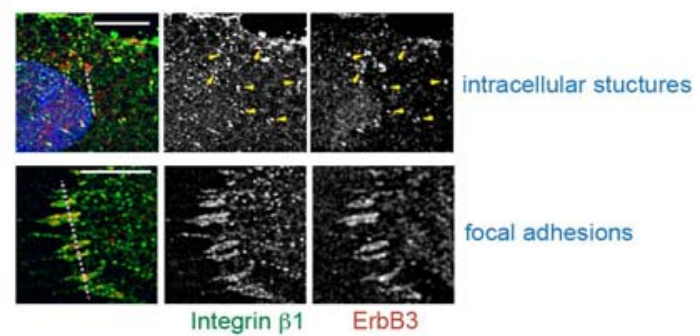

d

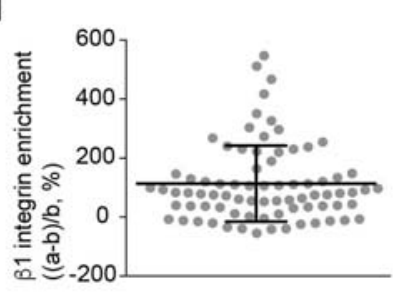

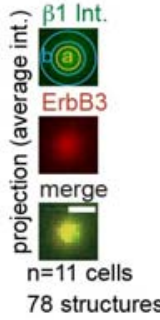
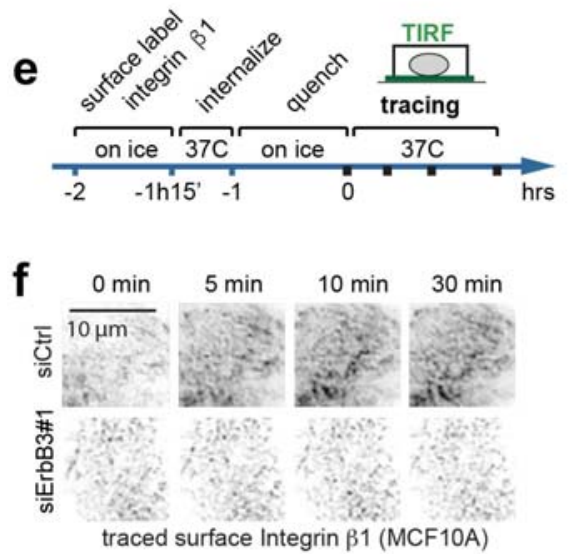

g

h
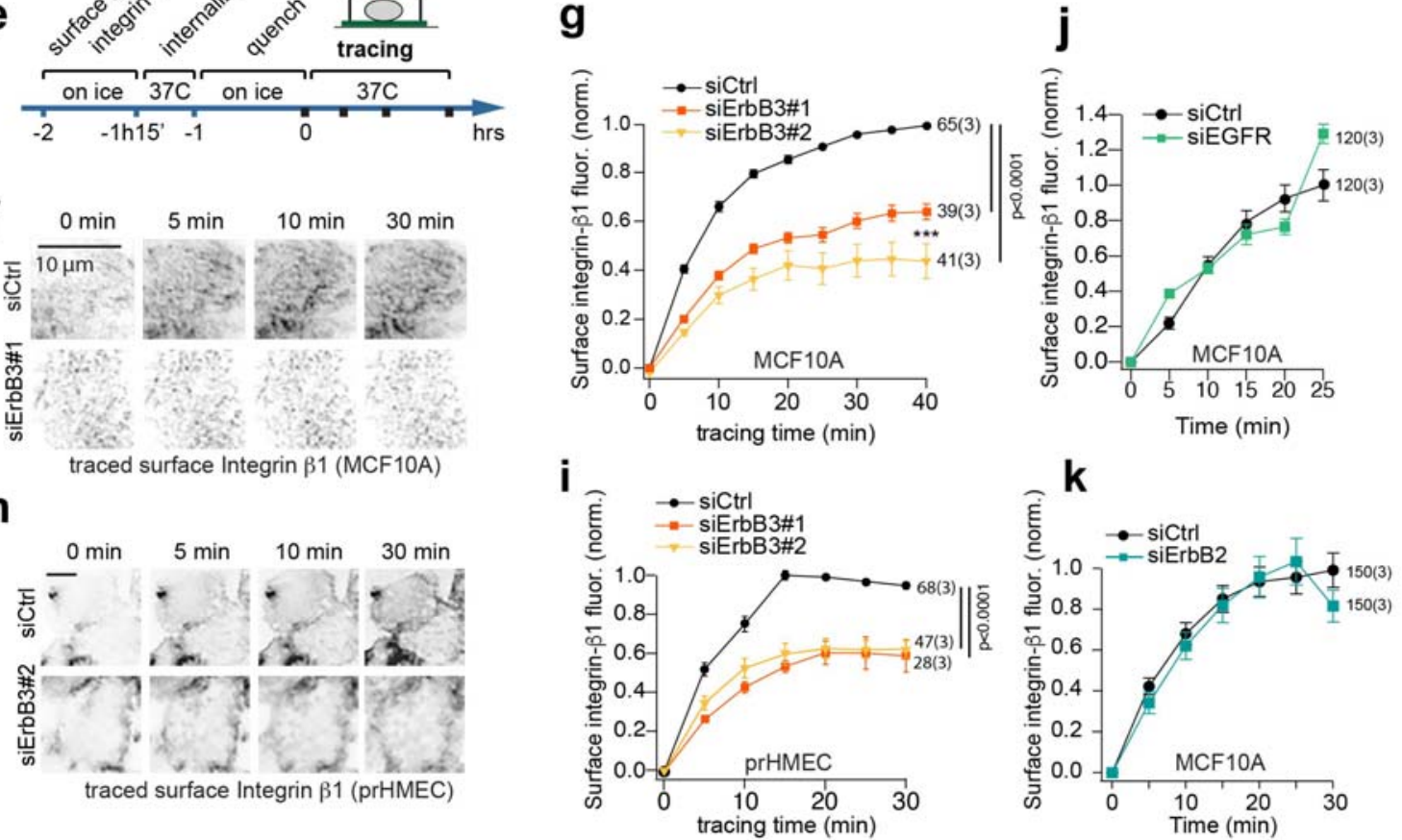

Figure 1: ErbB3, but not EGFR or ErbB2, promotes endocytic recycling of $\beta 1$ integrins. a-d

Confocal immunofluorescence imaging of traced surface labelled integrin $\beta 1$ and ErbB3: MCF7 cells were labelled on ice with an Alexa488-conjugated anti-integrin $\beta 1$ antibody prior to incubation for 30 minutes at $37^{\circ} \mathrm{C}$ to allow integrin $\beta 1$ internalisation, and subsequent cell-fixation and immunolabelling of ErbB3 (red) and counterstained with DAPI (blue). c Histogram of fluorescence intensities along dotted lines indicated in (b). d Analysis of colocalization of integrin $\beta 1$ and ErbB3. The enrichment 
Integral role of ErbB3 in the endocytic recycling machinery

of integrin $\beta 1$ in ErbB3-positive intracellular structures (0.5-2 $\mu \mathrm{m}$ diameter) was determined by the formula $(a-b) / b$ where $a$ is the integrin $\beta 1$ intensity at ErbB3 positive structures, and $b$ the adjacent intensity (background) for each structure. Average intensity projections of all analysed structures are shown on the right-hand side. e Schematic outline of the recycling assays conducted in (f-k) in the absence of growth factors: Briefly, the surface-pool of integrin $\beta 1$ was labelled with an Alexa488conjugated antibody and allowed to endocytose. Fluorophore label remaining on the cell surface was quenched with an anti-Alexa488 antibody, prior to visualisation of traced integrin $\beta 1$ re-emerging on the cell surface by live-cell TIRF microscopy. The recycling assays were conducted after prior transfection with non-targeting control siRNA or siRNA targeting indicated proteins. f Represenative TIRF microscopy images of integrin $\beta 1$ from peripheral areas of MCF10A cells. $g$ Quantifications of recycled integrin $\beta 1$ performed on indicated number of cells (outside of brackets on the right-hand side of graphs), from three independent experiments and shown as Alexa488 intensity normalized between $0-1$, with the control as reference where $F_{\text {norm }}=\left(\left(F_{\max }-F_{\min }\right) /\left(F-F_{\min }\right)\right)$. $\mathbf{h}$ Representative TIRF microscopy images of integrin $\beta 1$ from prHMEC cells. $\mathbf{i}$ Quantifications of recycled integrin $\beta 1$ in prHMECs performed as described in (g). j,k. Quantified recycling of integrin $\beta 1$, after prior siRNAmediated depletion of either EGFR (j) or ErbB2 (k). Data are presented as mean values \pm s.e.m. and Pvalues determined by two-tailed paired student's t-test. ns=non significant. Scale bar: $10 \mu \mathrm{m}$, except figure 1d (scale bar:1 $\mu \mathrm{m}$ ) 

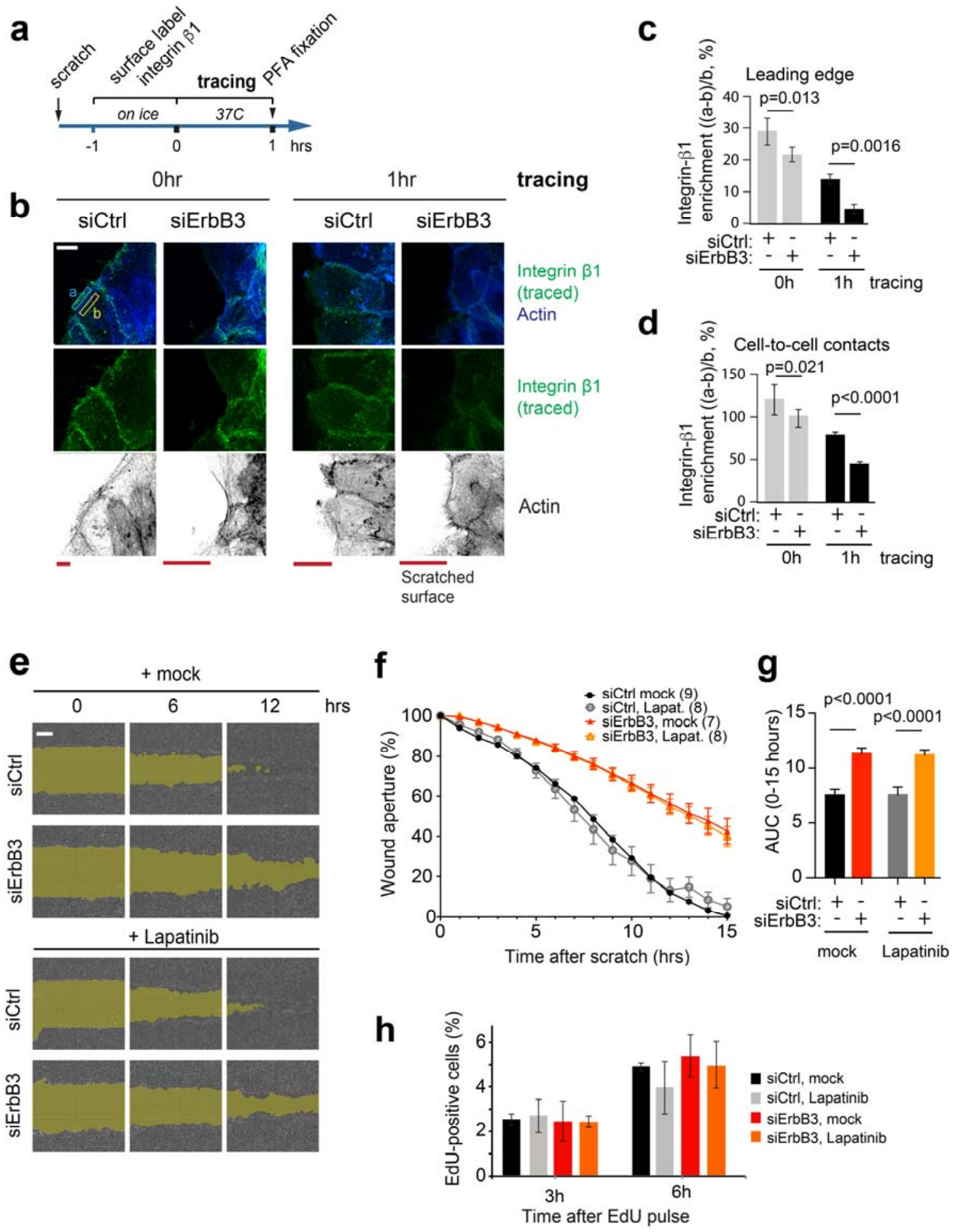

Figure 2: Ligand-independent role of ErbB3 in epithelial sheet motility. a-d Confocal immunofluorescence imaging of surface-labelled integrin $\beta 1$ (green) or actin (blue/black) on confluent sheets of MCF10A cells at $0 \mathrm{~h}$ or $1 \mathrm{~h}$ after labelling, as outlined in (a). Note in (b) that depletion of ErbB3 abrogates integrin $\beta 1$ localisation at the leading front. $\mathbf{c}$ Enrichment of integrin $\beta 1$ determined as $((a-b) / b)$ where $a=$ mean fluorescence intensity (integrin $\beta 1)$ at a defined area of the leading edge 
(c) or cell-cell contact (d) and b=mean intensity of adjacent cytoplasm of same area. Data are presented as mean values ( $>74$ cells per data point) \pm s.e.m., $n=3$ independent experiments. e Scratch closure assay of control or ErbB3-depleted MCF10A cells, cultured in serum-containing but growth factor-deprived media in the presence or absence of the EGFR/ErbB2 inhibitor Lapatinib. Wound area highlighted in yellow. f,g Quantification of scratch aperture (f) or area under curve, AUC, (g) of samples treated as in (e). Data are presented as mean values \pm s.e.m., n-values indicated in parenthesis. $\mathbf{h}$ Quantification of cell proliferation as incorporation of EdU for indicated times in control or ErbB3 siRNA-transfected cells in the presence or absence of $1 \mu \mathrm{M}$ lapatinib. Data are presented as mean values \pm s.e.m., $\mathrm{n}=3$ independent experiments. 


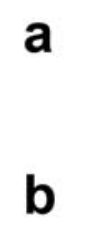

chase:

0

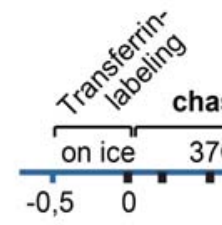

5
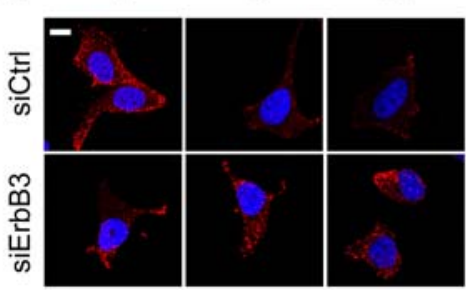

Transferrin-Alexa594, DAPI

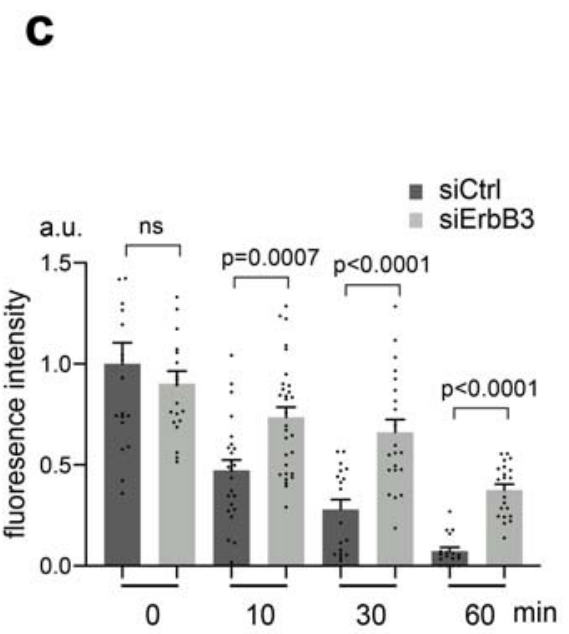

d

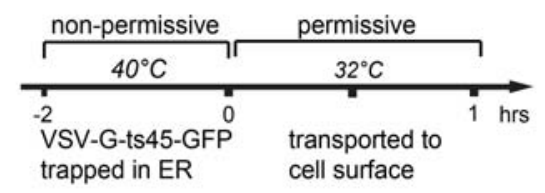

f

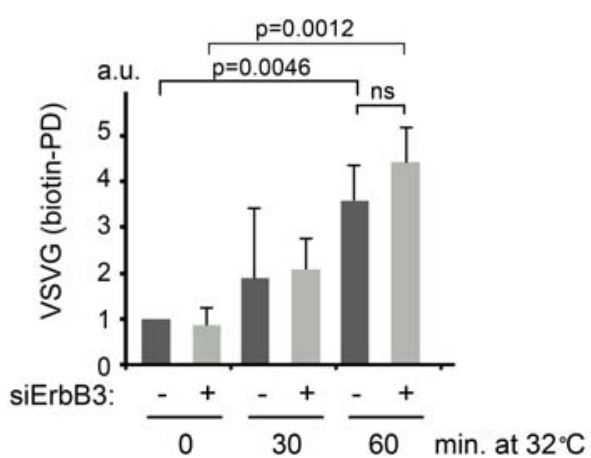

e
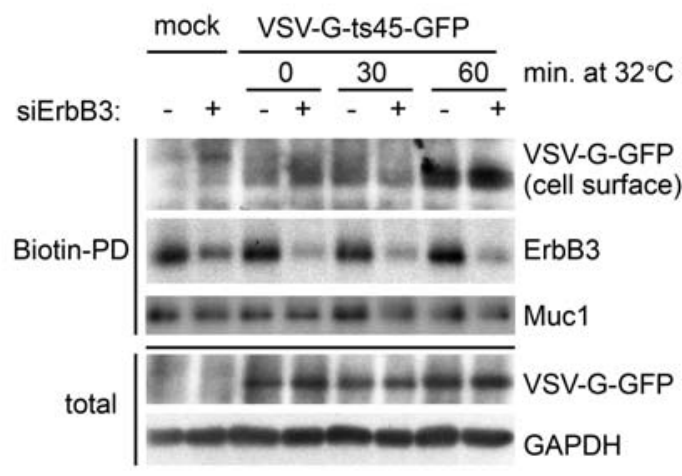

Figure 3: ErbB3 supports endocytic recycling of the transferrin receptor but not secretory

trafficking of VSV-G. a Confocal imaging of Alexa594-conjucated transferrin chased with unlabelled holo-transferrin for indicated times in MCF7 cells. Note that siRNA-mediated depletion of ErbB3 caused prolonged intracellular retention of transferrin. b Quantification of Alexa594 fluorescence intensity in cells treated as in $\mathbf{a}$ ( $\mathrm{n}>17$ cells for each data point from three experiments) normalised against the control siRNA treated, 0 hour timepoint of each independent experiment. b,d,f Data are presented as mean values \pm s.e.m. $P$ values determined by two-tailed paired student's t-test. Scale bar: $10 \mu \mathrm{m}$. $\mathbf{c}$ experimental outline of the VSVG trafficking experiments (d and e). d,e Western blot analysis of the surface pool of VSV-G-ts45-GFP (pulldown of surface-biotinylated VSV-G-ts45GFP), after its release from the endoplasmic reticulum (ER) at permissive temperature for indicated times. Note that ErbB3-depletion did not influence secretive trafficking of VSVG from the ER. $\mathbf{e}$ quantification of normalised levels of VSV-G-GFP in biotin-pulldowns as determined by immunoblot band intensities ( $n=3$ independent experiments). Data are presented as mean values \pm s.e.m. $P$ determined by two-tailed paired student's t-test. ns=non significant. 
a

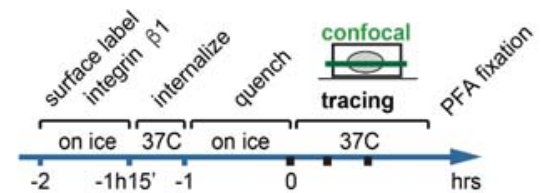

b

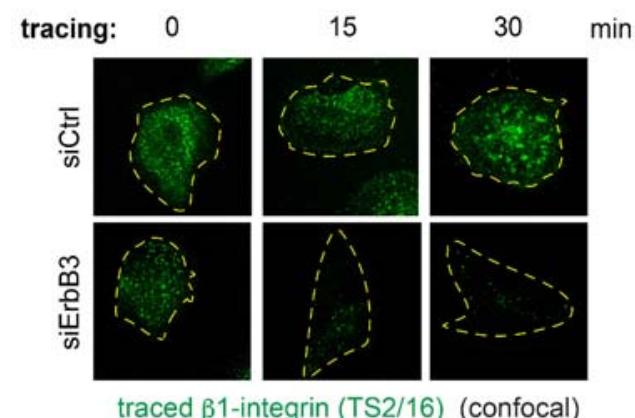

d
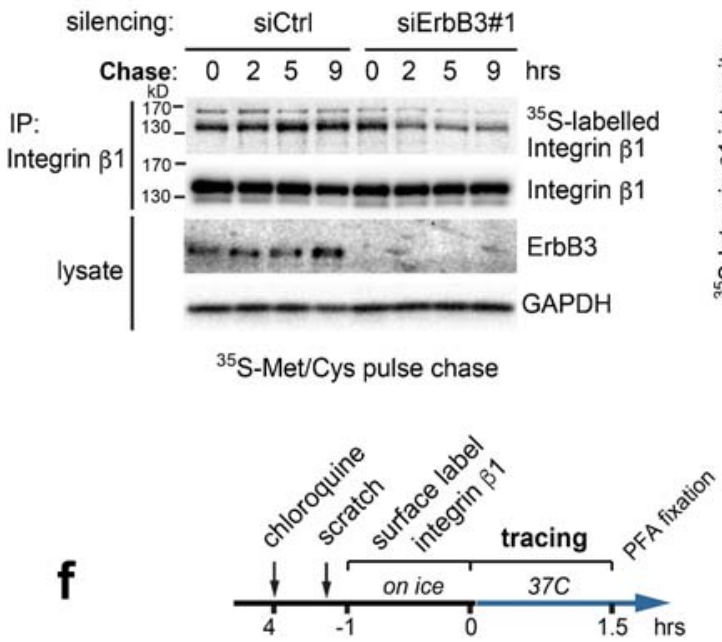

g

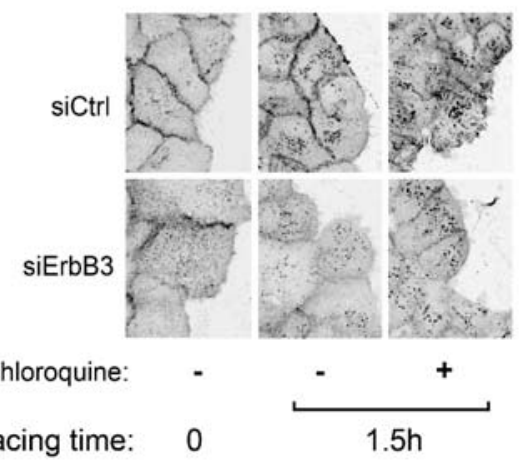

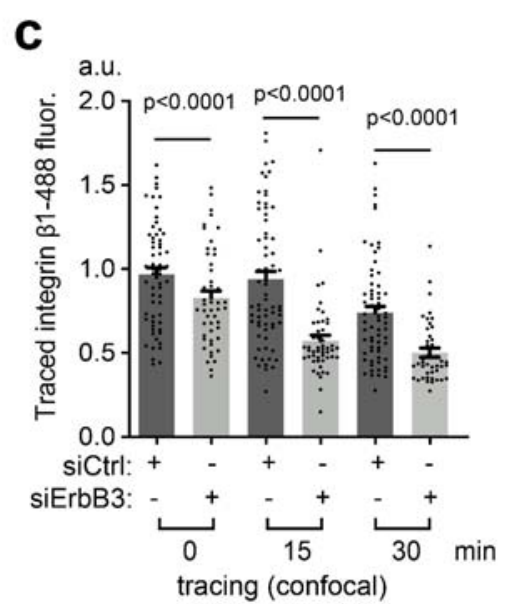

e
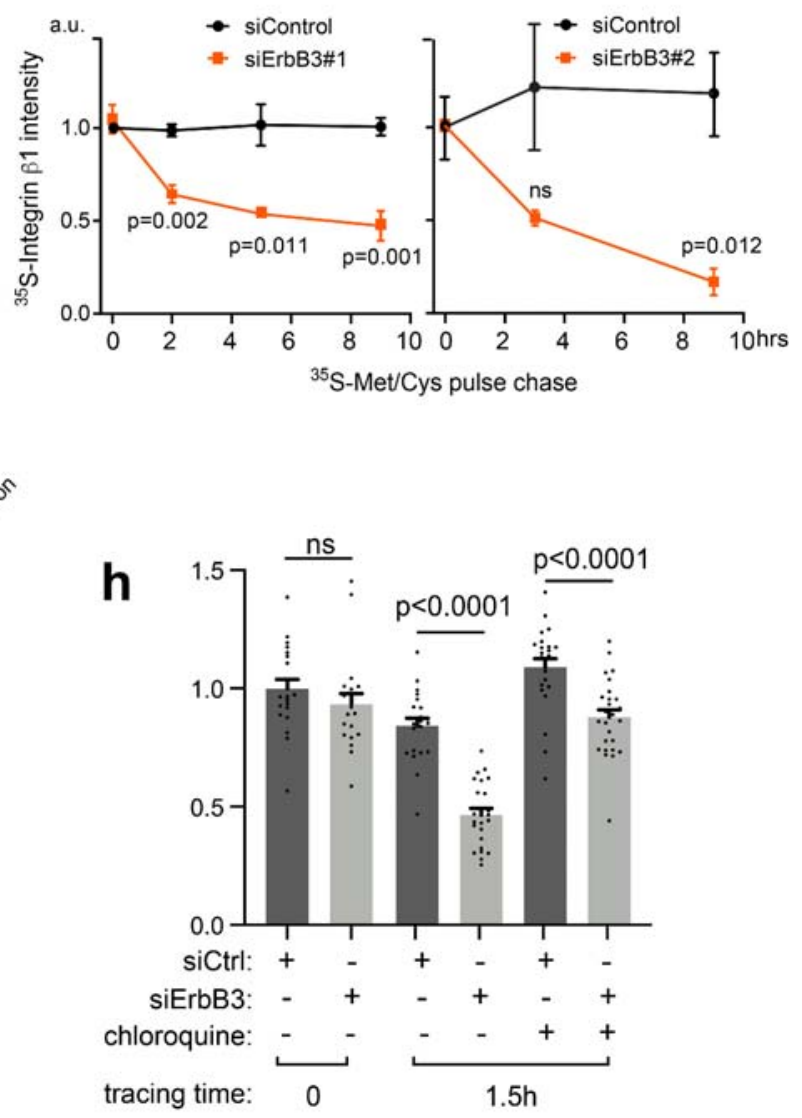

Figure 4: Loss of ErbB3 directs Integrin $\beta 1$ towards lysosomal degradation. a,b Confocal immunofluorescence imaging of traced internalised integrin $\beta 1$ : The MCF10A cells were transfected with control or ErbB3 siRNA and assay performed in growth factor deprived media. c Quantification of immunofluorescence intensity of internalised integrin $\beta 1$ traced for indicated times $(n>32$ cells per data point from 5 independent experiments). d Determination of integrin $\beta 1$ turnover by pulse-chase 
Integral role of ErbB3 in the endocytic recycling machinery

metabolic labelling: Control or ErbB3 siRNA-transfected MCF10A cells were pulse-chase labelled with radioactive $\left({ }^{35} \mathrm{~S}\right)$ methionine and cysteine. Radiolabelled integrin $\beta$ was visualised by radiography of immunoprecipitates (upper panel). Cell lysates and immunoprecipitates were analysed by immunoblotting. e Quantification of pulse chased ${ }^{35}$ S-labelled integrin $\beta 1$, as in (d) $(n=4$ independent experiments). f,g Confocal immunofluorescence imaging of surface-labelled integrin $\beta 1$ (using an Alexa488-conjugated anti-integrin $\beta 1$ antibody), prior to (0 hours) or after tracing at $37^{\circ} \mathrm{C}$ for 1.5 hours. A scratch was inflicted prior to antibody incubation. Note that application of the lysosome inhibitor chloroquine caused accumulation of integrin $\beta 1$ in intracellular vesicular compartments both in control of ErbB3-depleted cells. h Quantification of integrin $\beta 1$ fluorescence intensity in cells bordering the migratory front in samples treated as in $\mathbf{g}$, showing that chloroquine restored levels of integrin $\beta 1$ in ErbB3-depleted cells. Data presented as mean values \pm s.e.m., $n=19-27$ cells per data point from 3 independent experiments. $\mathrm{P}$ values determined by two-tailed paired student's t-test. ns=non-significant. 


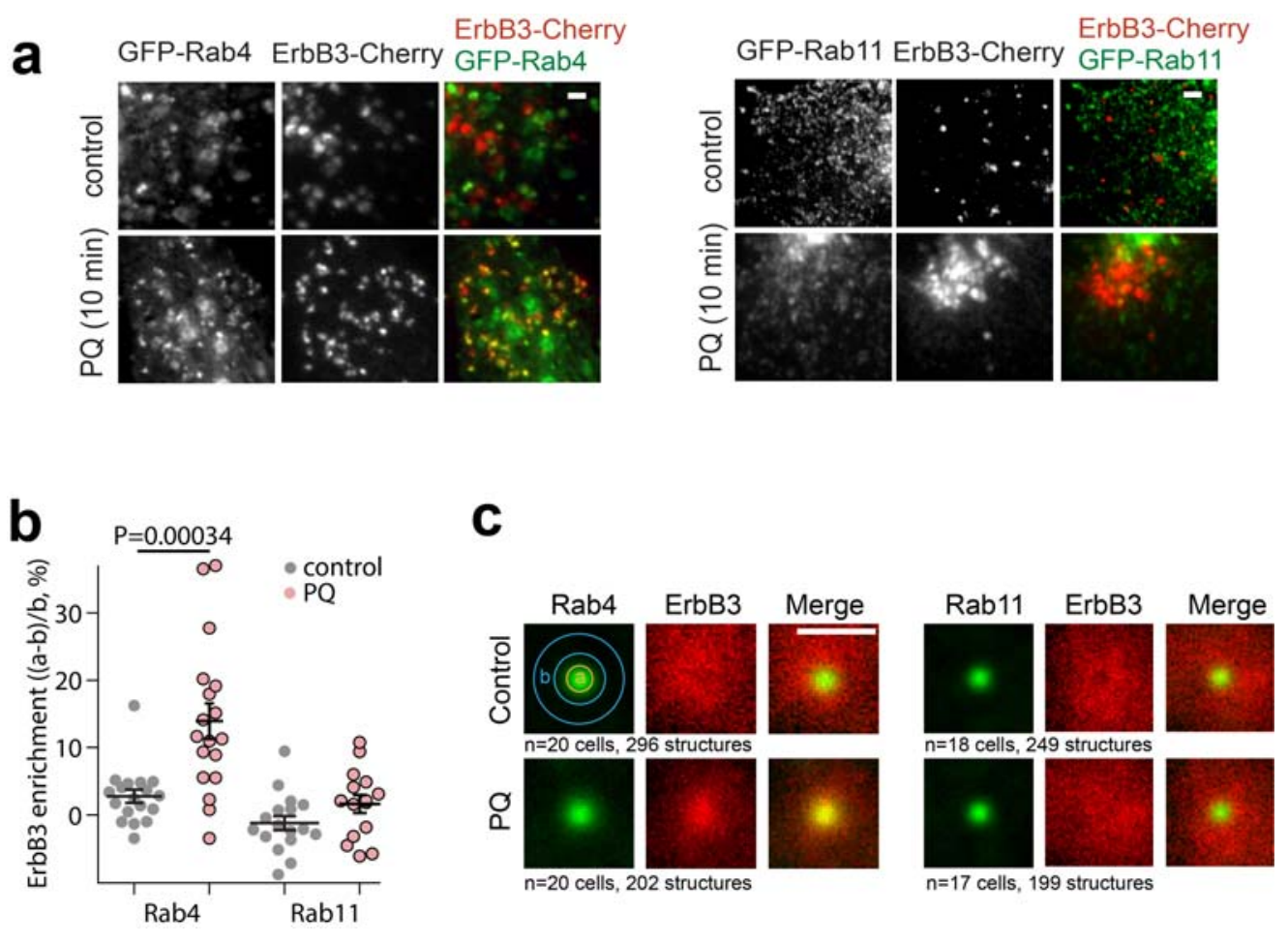

Figure 5: ErbB3 co-localises with Rab4-positive vesicles. a Confocal imaging of ErbB3-mCherry and indicated Rab marker expressed in MCF7 cells, with or without prior treatment with the recycling inhibitor primaquine (PQ). b Analysis of colocalization of ErbB3-mCherry and Rab4 or Rab11. The relative enrichment of ErbB3 at the Rab-positive structures was determined by the formula (a-b)/b where $a$ is the ErbB3-mCherry intensity of the center of Rab4 structures, and b the adjacent volume (background) for each structure. Each data point represents the average of a minimum of 20 structures in one cell. P-values were determined using unpaired, 2-tailed Student's t-test. c Average projections of all analysed (indicated number) of GFP-Rab4 or GFP-Rab11 positive structures from indicated number of cells (3 independent experiments). 
a

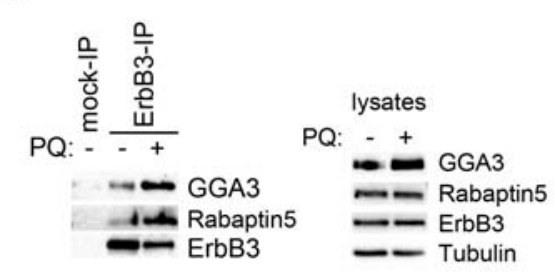

b

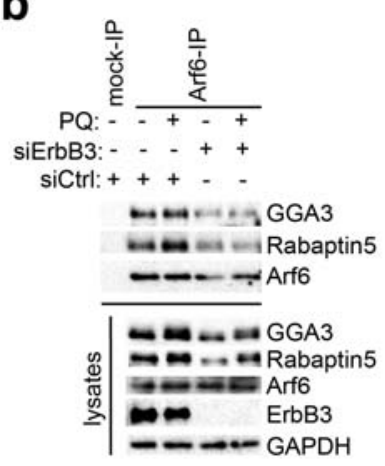

C
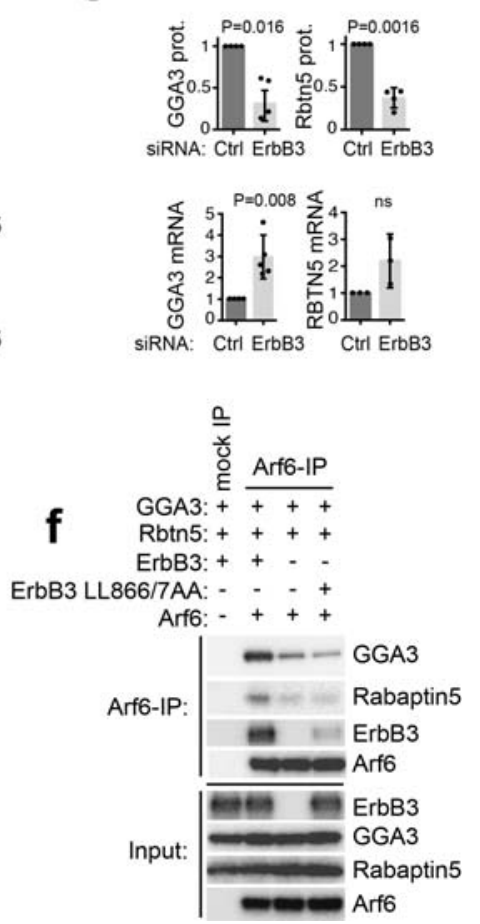

Figure 6: ErbB3 scaffolds assembly of the Arf6-GGA3-Rabaptin5 endosomal sorting complex. a Immunoblotting of ErbB3 immunoprecipitates or input cell lysates, after 30 minutes treatment with primaquine (PQ), showing endogenous binding of ErbB3 with GGA3 and Rabaptin5 that increases upon PQ treatment, and the presumed accumulation of recycling endosomes (representative of 3 independent experiments). b Immunoblotting of Arf6 immunoprecipitates or input cell lysates, following control or ErbB3 siRNA transfection and 10 minutes treatment with PQ or vehicle. Note that endogenous co-precipitation of Arf6 with both GGA3 and rabaptin5 is reduced in the absence of ErbB3. c Quantification of GGA3 and Rabaptin5 protein levels (by western blotting) and mRNA levels (by quantitative RT-PCR) in ErbB3 siRNA-transfected MCF10A cells relative to controltransfected cells ( $n=4$ experiments for protein and $n=3$ for mRNA). Data are presented as mean values \pm s.e.m. and $\mathrm{P}$ values (one sample student's t-test). d Structural model highlighting the putative GGA3binding motif 864-DxxLL-867 in the ErbB3 kinase domain. e Immunoblotting of ErbB3 immunoprecipitates or input cell lysates, after ectopic expression of ErbB3 or the ErbB3 LL866/867AA mutant with GGA3 in HEK293T cells. Note that the LL866/867AA mutant ErbB3 fails to co-precipitate with GGA3. f The LL866/867AA mutation compromises the ability of ErbB3 to promote assembly of the Arf6-GGA3-Rabatin5 sorting complex: Immunoblotting of Arf6 immunoprecipitates or input cell lysates, following ectopic expression of Arf6, GGA3 and Rabaptin5 (Rbtn5), with or without ErbB3 or ErbB3-LL866/867AA. 
a

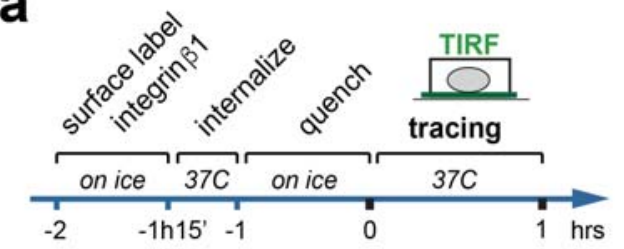

b

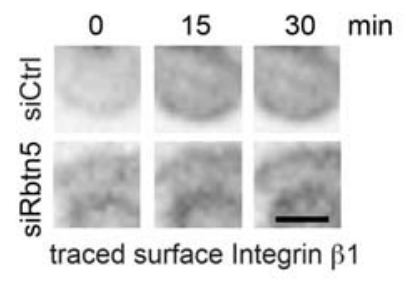

e

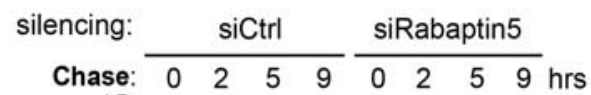

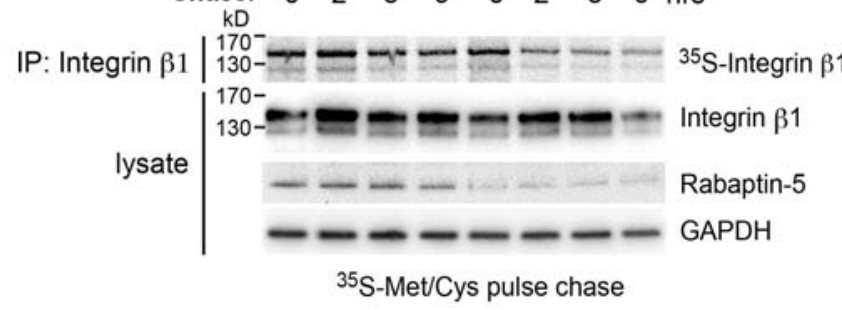

C

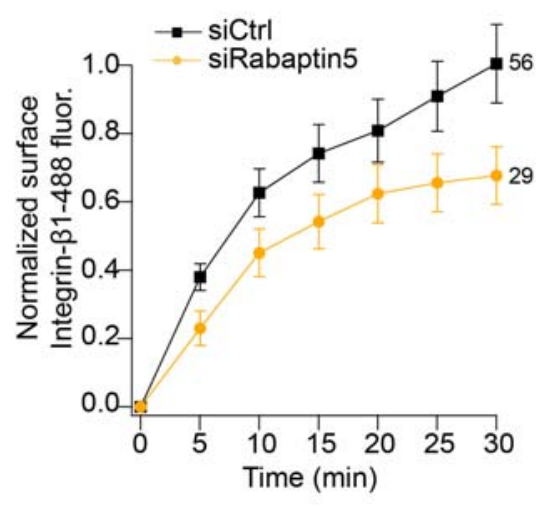

d

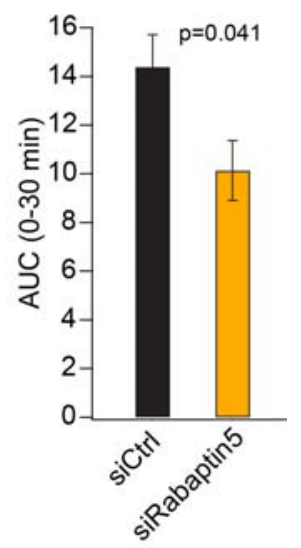

Figure 7: Loss of Rabaptin5 reduces integrin $\beta 1$ recycling and promotes its degradation. a

Schematic outline of the recycling assay performed in b-d. b-d Re-emergence of traced internalised integrin $\beta 1$ on the cell surface was visualized by live-cell TIRF imaging, after prior transfection of control or Rabaptin5-targeting siRNA. c Quantification of recycled integrin $\beta 1$ was conducted on indicated number of cells (right-hand side of graphs) from three independent experiments and shown as Alexa488 intensity normalized against levels at onset of tracing (0) and maximum intensity (1). d Columns show area under curves (AUC). e Determination of integrin $\beta 1$ turnover by pulse-chase metabolic labelling. Control or rabaptin5 siRNA-transfected MCF10A cells were pulse-chase labelled with radioactive $\left({ }^{35} \mathrm{~S}\right)$ methionine and cysteine. Radiolabelled integrin $\beta 1$ was visualised by radiography of integrin $\beta 1$ immunoprecipitates (upper panel). Cell lysates and immunoprecipitates were analysed by immunoblotting as indicated. $\mathbf{f}$ Quantification of radiolabelled integrin $\beta 1$ at the indicated times of three independent pulse and chase experiments. Data are presented as mean values \pm s.e.m. $\mathrm{P}$ values determined by two-tailed paired student's t-test. ns=non significant. 
Integral role of ErbB3 in the endocytic recycling machinery

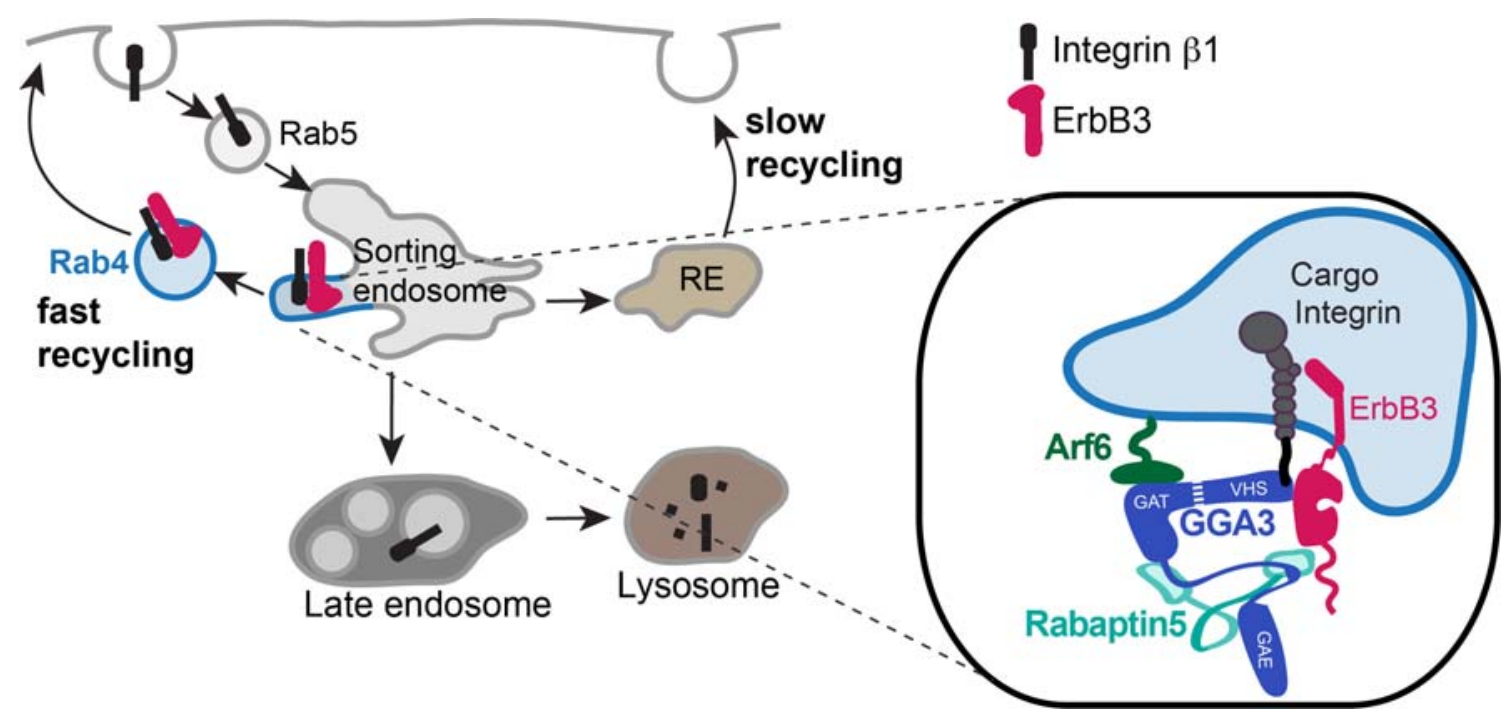

Figure 8: ErbB3 sorts cargo for endocytic recycling. Proposed model: ErbB3 promotes endocytic recycling by scaffolding the Arf6-GGA3-rabaptin5 endocytic sorting complex to sort cargo, including $\beta 1$ integrins and TfR for Rab4-dependent recycling. 
a

C

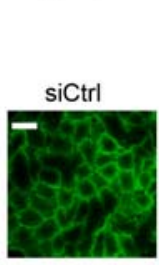

MCF10A

e

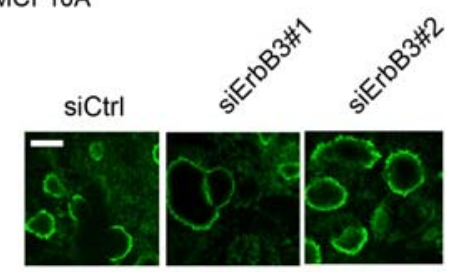

prMEC
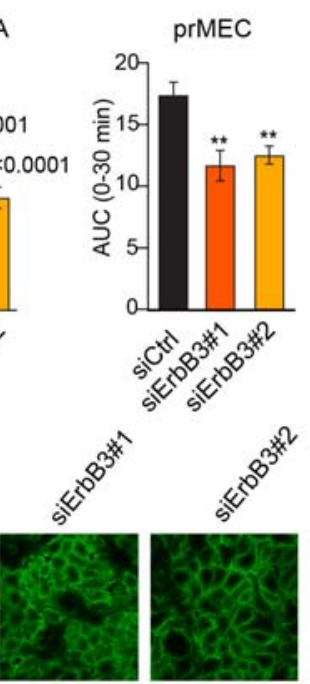

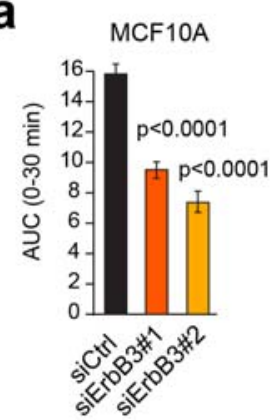

b

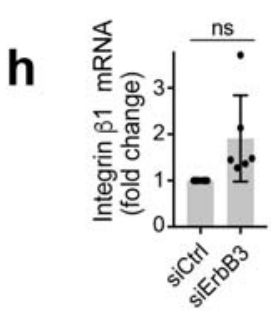

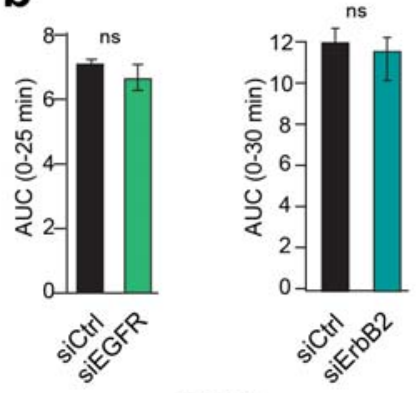
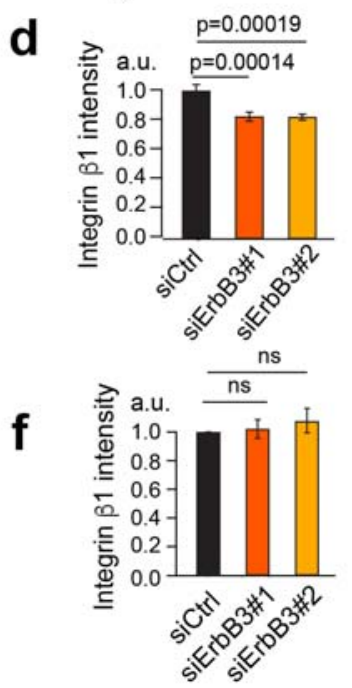

g

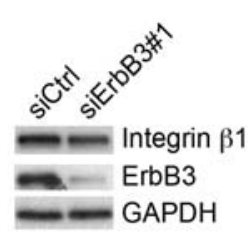

siErbB2

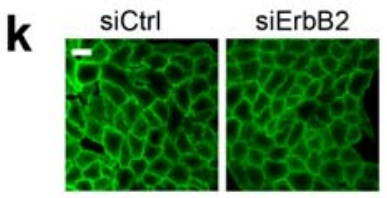

MCF10A

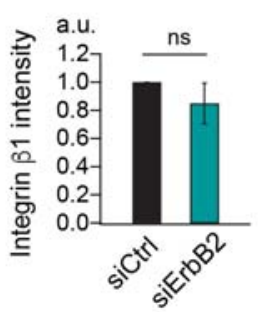

i

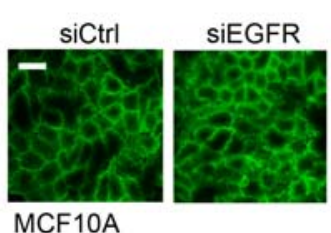

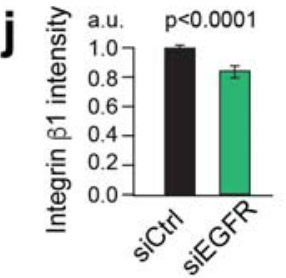

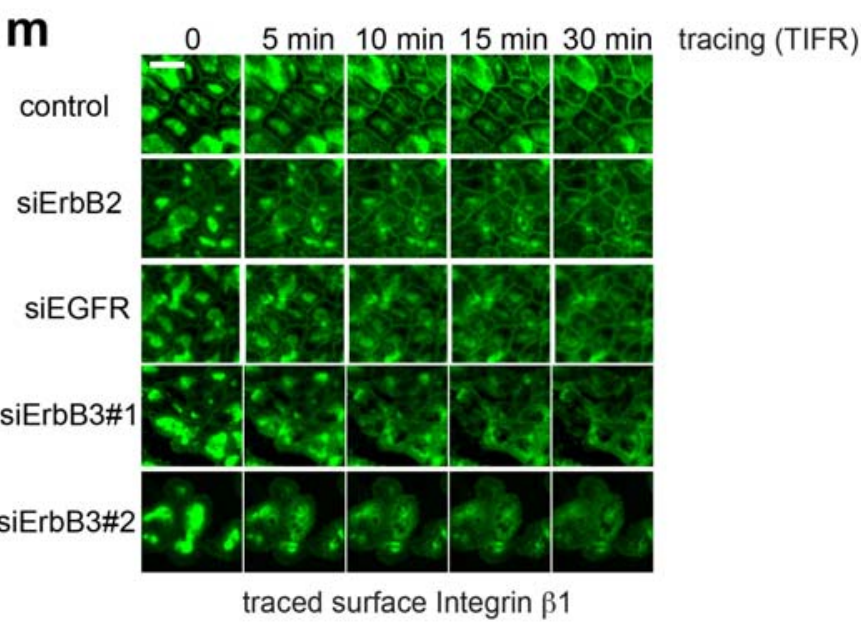

Supplementary Figure 1: ErbB3, but not EGFR or ErbB2, promotes endocytic recycling of $\beta 1$ integrins. a Related to figures 1g,i: Columns show area under curve (AUC) of the $\beta 1$ integrin 
recycling data (figures $1 \mathrm{~g}, \mathrm{i}$ ). b Related to figures $1 \mathrm{j}, \mathrm{k}$ : shows AUC of the $\beta 1$ integrin recycling data (figures $1 \mathrm{j}, \mathrm{k}$ ). Data presented as mean values \pm s.e.m. n-values indicated in main figures. c-f Immunofluorescence imaging of the surface pool of integrin $\beta 1$ labelled with Alexa488-conjugated antibody on ice for 1 hour, on MCF10A (c,d) or prMEC cells $(e, f)$ transfected with control or ErbB3targeting siRNAs. Columns (d,f) show quantification of integrin $\beta 1$ fluorescence intensity, normalised against control siRNA treated samples. Data presented as mean values \pm s.e.m. n-values indicated in main figures (figures $1 \mathrm{j}, \mathrm{k}$ ). Note that ErbB3-depletion does not significantly influence surface levels of $\beta 1$ integrins under experimental conditions representing onset of the tracing experiments, prior to quenching. (Fig. 1f-i). g Western blot analysis of ErbB3 and integrin $\beta 1$ after transfection of MCF10A cells with control or ErbB3 siRNA. h Relative levels of ErbB3 mRNA in control or ErbB3 siRNA transfected cells as determined by quantitative PCR ( $n=6$ independent experiments). Data presented as mean \pm s.d., from 6 independent experiments. ns=non-significant, as determined by one-sample t-test. i-l Immunofluorescence imaging of the surface pool of integrin $\beta 1$ labelled with Alexa488-conjugated antibody on ice for 1 hour, on MCF10A cells transfected with control siRNA or siRNAs targeting $\operatorname{EGFR}(i, j)$ or ErbB2 $(k, l)$. j,l Quantification of integrin $\beta 1$ fluorescence intensity, normalised against control siRNA-treated samples. Data presented as mean values \pm s.e.m. $\mathbf{m}$ Live-cell TIRF imaging of MCF10A cells to monitor recycling of labelled integrin $\beta 1$, after prior siRNA-mediated depletion of EGFR, ErbB2 or ErbB3. Experiments $\mathbf{c}$ and $\mathbf{i}$ were performed at the same time therefore the control images are the same. 


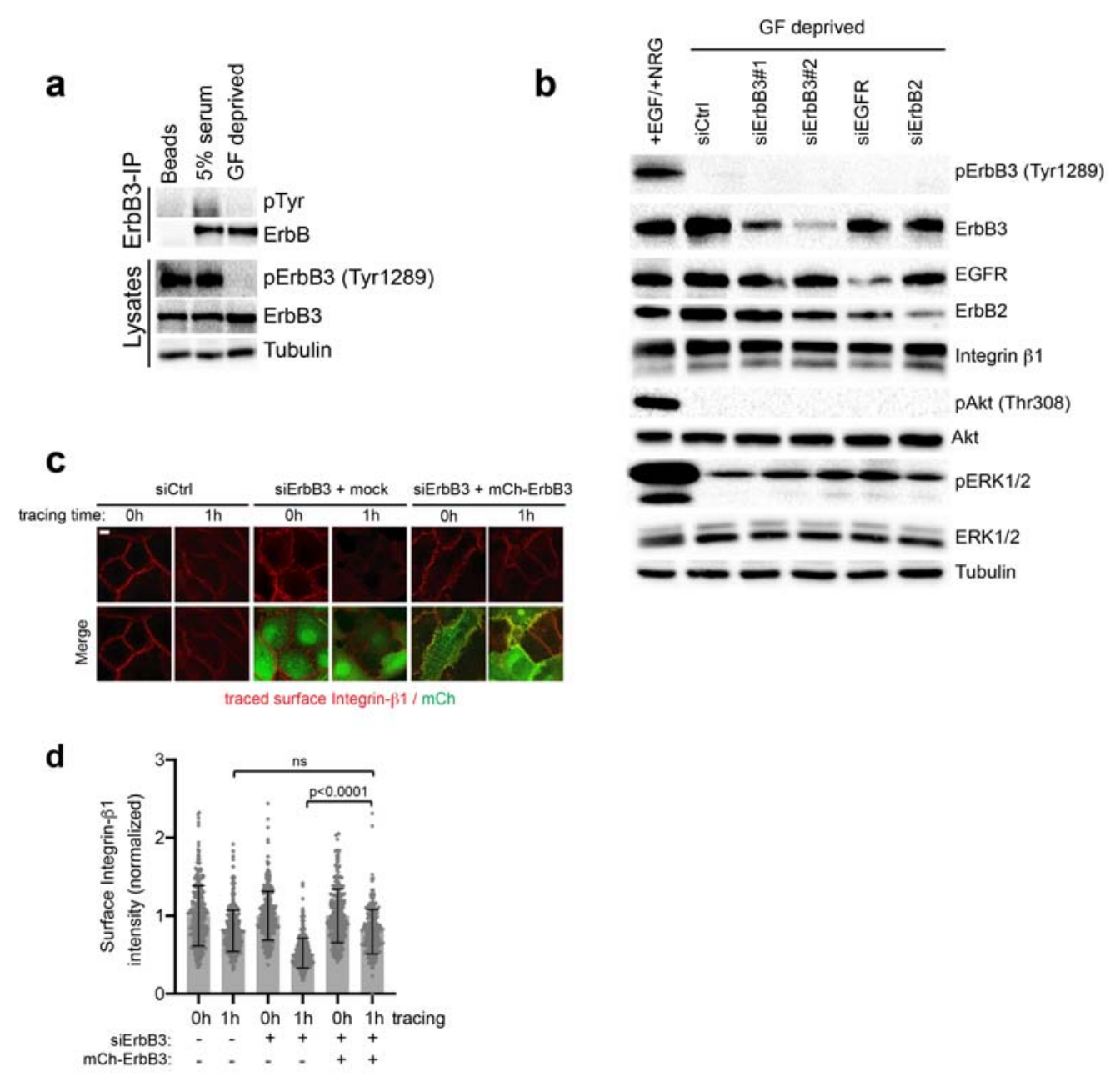

Supplementary Figure 2: ErbB3 promotes endocytic recycling of $\beta 1$ integrins in absence of detectable tyrosine-kinase signalling. a Western blot analysis of ErbB3 immunoprecipitates or total cell lysates of MCF10A cells cultured in the presence of 5\% horse serum or under growth factor (GF) deprived conditions. Antibodies detecting total phospho-tyrosine (4G10; pTyr) or the specific phospho-tyrosine 1289 on ErbB3 were used. b Western blot analysis of total MCF10A cell lysates under GF deprived culture conditions or upon supplementation of the EGF and $\beta N R G$, after depletion of EGFR, ErbB2 or ErbB3. c,d Expression of siRNA-resistant ErbB3 restores the surface pool of traced $\beta 1$ integrins in ErbB3-depleted MCF10 cells: Confocal imaging of integrin $\beta 1$ labelled on the cell surface with an Alexa488-conjugated antibody, prior to (0h) or after tracing (1h), on cells expressing siRNA-resistant mCh-ErbB3 or fluorophore alone with or without transfection of control or ErbB3 siRNA as indicated. Fluorescence-intensity (integrin $\beta 1$ ) along cell-cell borders was quantified from 3 independent experiments. Values were normalised against intensities prior to tracing (0h). The results suggest that off-target effects of the ErbB3 siRNAs do not underlie the observed recycling defect. Data presented as mean values \pm s.d., $n>230$ cell-cell borders from 3 independent experiments and $\mathrm{p}$ values determined by two-tailed paired student's t-test. 
a

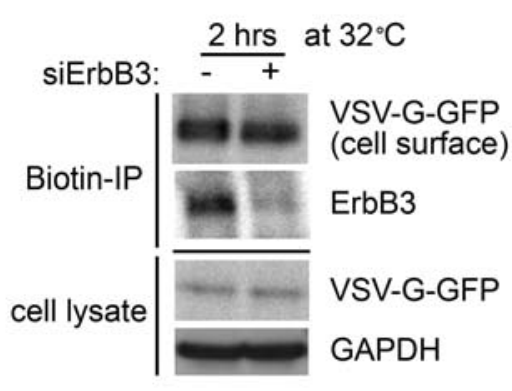

b $\quad 40^{\circ} \mathrm{C}$

0

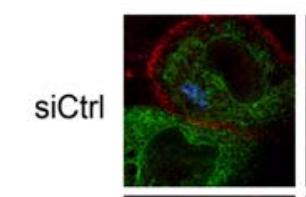

siErbB3
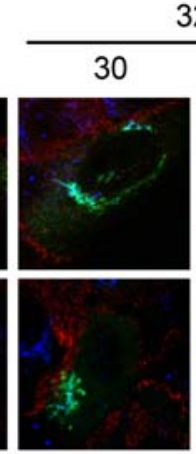

$32^{\circ} \mathrm{C}$ $60 \quad \min$
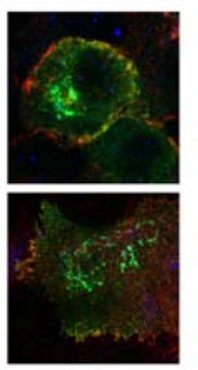

VSV-G-ts45-GFP / GM130 / E-cadherin

Supplementary Figure 3: ErbB3 does not regulate exocytic trafficking of VSVG from the ER. a

Western blot analysis of isolated surface-biotinylated VSV-G-ts45-GFP, following its temperaturedependent release from the endoplasmic reticulum (ER) 2 hours prior. Note that ErbB3-depletion does not influence the emergence of VSVG at the plasma membrane. b Confocal imaging of VSV-G-ts45GFP and markers of the TGN (GM130) and the cell surface (E-cadherin) in control or ErbB3 siRNA transfected MCF7 cells at non-permissive temperature (40C) and following its subsequent shift to permissive (32C) temperature. Note that VSV-G-ts45-GFP localises to the ER at 40C, while after 30 minutes at permissive temperature most of the VSV-G-ts45-GFP has moved to the TGN and after 1 hour to the cell surface, both in the presence or absence of ErbB3.

a

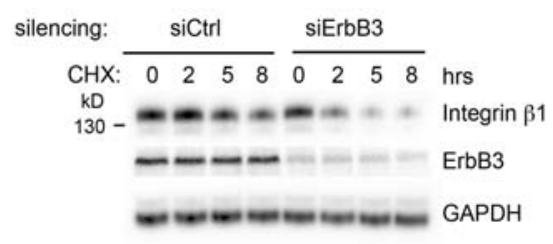

b

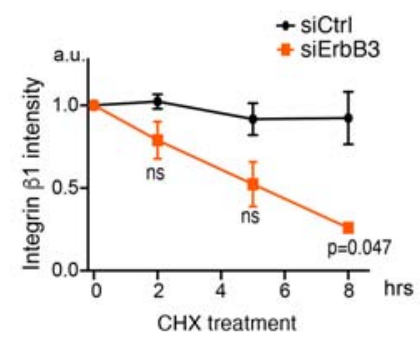

\section{Supplementary Figure 4: integrin $\beta 1$ stability is reduced upon depletion of ErbB3.}

a Western blotting analysis of MCF10A cells, transfected with control or ErbB3 siRNA prior to treatment with either protein synthesis inhibitor cycloheximide (CHX) or vehicle alone for indicated times. b Quantification of integrin $\beta$ band intensities presented as mean values \pm s.e.m., $n=3$ independent experiments and $\mathrm{p}$ values determined by two-tailed paired student's t-test. 


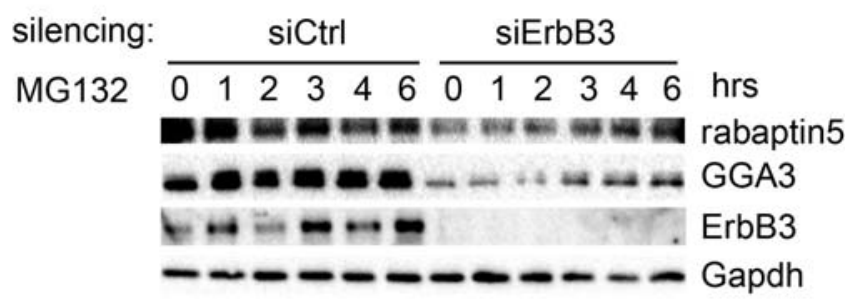

b

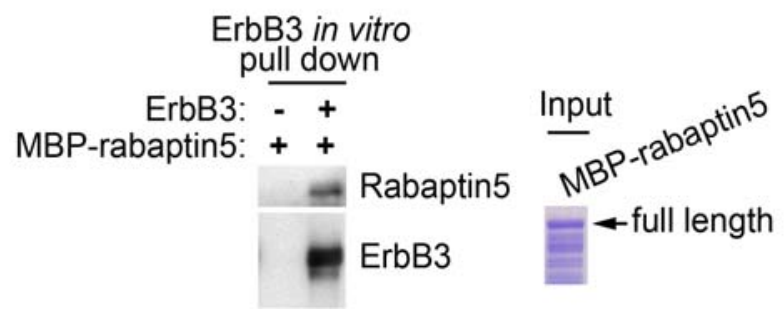

Supplementary Figure 5: a ErbB3 binds Rabaptin5 in vitro and promotes its stability. Western blotting analysis of MCF10A cells, transfected with control or ErbB3 siRNA prior to treatment with the proteasome inhibitor MG132 for indicated times. MG321 partially restores GGA3 and Rabaptin5 levels in ErbB3-depleted cells. b In vitro binding of ErbB3 to rabaptin5. Western blotting of ErbB3immunoprecipitates following incubation of recombinant ErbB3 and rabaptin5. 